\title{
Interactions between aquatic plants and turbulent flow: a field study using stereoscopic PIV
}

\author{
S. M. Cameron $\dagger$, V. I. Nikora, I. Albayrak, O. Miler, M. Stewart \\ and F. Siniscalchi \\ School of Engineering, University of Aberdeen, Kings College, Aberdeen AB24 3UE, UK
}

(Received 10 December 2012; revised 22 July 2013; accepted 4 August 2013; first published online 5 September 2013)

A stereoscopic particle image velocimetry (PIV) system for use in shallow $(\sim 0.5 \mathrm{~m}$ deep) rivers was developed and deployed in the Urie River, Scotland, to study the interactions between turbulent flow and a Ranunculus penicillatus plant patch in its native environment. Statistical moments of the velocity field were calculated utilizing a new method of reducing the contribution of measurement noise, based on the measurement redundancy inherent in the stereoscopic PIV method. Reynolds normal and shear stresses, their budget terms, and higher-order moments of the velocity probability distribution in the wake of the plant patch were found to be dominated by the presence of a free shear layer induced by the plant drag. Plant motion, estimated from the PIV images, was characterized by travelling waves that propagate along the plant with a velocity similar to the eddy convection velocity, suggesting a direct coupling between turbulence and the plant motion. The characteristic frequency of the plant velocity fluctuations $(\sim 1 \mathrm{~Hz})$ may suggest that the plant motion is dominated by large eddies with scale similar to the flow depth or plant length. Plant and fluid velocity fluctuations were, in contrast, found to be strongly correlated only over a narrow $(\sim 30 \mathrm{~mm})$ elevation range above the top of the plant, supporting a contribution of the shear layer turbulence to the plant motion. Many aspects of flow-aquatic plant interactions remain to be clarified, and the newly developed stereoscopic field PIV system should prove valuable in future studies.

Key words: flow-structure interactions, river dynamics, shear layer turbulence

\section{Introduction}

Aquatic plants play a vital role in the management and healthy functioning of river ecosystems. They provide habitat, refuge, and food for periphyton, invertebrates, and fish; they produce oxygen and sink carbon through photosynthesis; they regulate sediment transport and mixing, and they contribute to hydraulic resistance (e.g. Naden et al. 2006; Bornette \& Puijalon 2011; Folkard 2011b; Nepf 2012). Understanding of these processes is important for the successful management of river systems (mitigating flood risk, preserving biodiversity, maintaining water quality) but is still limited by a lack of fundamental knowledge of the interactions between plants and 
flowing water. One of the reasons for this is that flow-plant interactions are scale dependent, covering a wide range of scales from the sub-leaf to the plant patch and larger scales, and are thus controlled by several complex and interlinked phenomena such as turbulence, viscous and pressure drag forces, plant biomechanical properties, and plant motion (Nikora 2010). Another reason is that experimental measurement of these phenomena remains challenging. Many of the experimental investigations on aspects of flow-plant interactions have been carried out in laboratory flumes using artificial plant replicas or plant surrogates (e.g. Ghisalberti \& Nepf 2002; Nezu \& Sanjou 2008; Siniscalchi, Nikora \& Aberle 2012), or using real plants attached to the bed in some artificial way (e.g. Sand-Jensen 2003; O'Hare, Hutchinson \& Clarke 2007; Siniscalchi \& Nikora 2012). Although these studies allow systematic manipulation of flow conditions and deployment of a full array of experimental technologies, it remains an open question as to whether they are truly representative of real plants in their natural habitats. A number of field studies have also been carried out (e.g. Koehl \& Alberte 1988; Sand-Jensen \& Mebus 1996; Green 2005; Naden et al. 2006; Sukhodolova \& Sukhodolov 2012). These studies, however, inevitably resort to point velocity measurement techniques (often involving only time-averaged velocities) which miss much of the detailed structure of the flow field.

In the study reported here, the need for more extensive field data on flow-aquatic plant interactions is addressed by developing a stereoscopic particle image velocimetry (PIV) system for field use and deploying it in the Urie River, Scotland. The PIV technique has been previously used outside of a laboratory (e.g. Nimmo Smith et al. 2002; Zhu et al. 2006; Tritico, Cotel \& Clarke 2007; Katija \& Dabiri 2008; Liao et al. 2009). This study, however, is the first time the stereoscopic PIV method has been used in the field, allowing all three components of the velocity vector to be captured. The system is utilized to study the interactions between river turbulence and the motion of a Ranunculus penicillatus plant patch in its natural environment.

The structure of the paper is as follows. First, the design of a stereoscopic PIV system that can be deployed in small rivers $(\sim 0.5 \mathrm{~m}$ flow depth) is discussed, including system calibration, analysis algorithms, and a new method of reducing the contribution of measurement errors to certain velocity statistics. Second, features of the field site selected for the study are identified, and measurement errors are analysed. Third, statistics of the flow field in the wake of the Ranunculus plant patch are evaluated, including terms of the Reynolds stress budget equation, spectra, and convection velocity. Fourth, statistics of the plant motion are evaluated along with correlations between turbulence and plant movement. Finally, potential interaction mechanisms between the plants and the flow are discussed.

\section{In-situ stereoscopic PIV system}

The in-situ stereoscopic PIV system was designed to utilize existing components from a custom-made laboratory PIV system including the laser (Oxford Lasers NanoL-50/100 PIV, twin Nd:YAG, $100 \mathrm{~mJ}$ at $50 \mathrm{~Hz}$ ) and cameras (Dalsa 4M60, CMOS, $2352 \times 1728$ pixels at 60 frames per second, $7.4 \mu \mathrm{m}$ pixel pitch, $60 \%$ effective fill factor, $532 \mathrm{~nm}$ bandpass optical filter, $60 \mathrm{~mm}$ lens at $\mathrm{f} / 5.6$ ) and direct-to-disk image recording setup $(4 \times 7200$ r.p.m. SATA disks in RAID 0 per camera $)$. At the core of the design is a glass-bottomed boat-shaped structure which sits at the water surface and allows a pair of cameras and the laser light sheet stable optical access through the fluctuating water surface of the river (figure 1). The streamlined design of the 'boat' limits the disturbed region of the flow field to a thin boundary layer near the water 
(a)

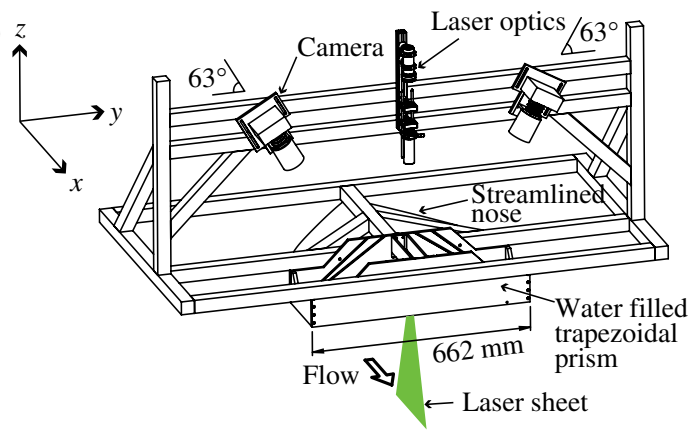

(b)

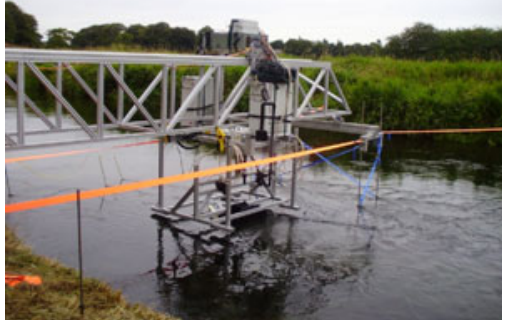

FIGURE 1. (Colour online) (a) Schematic of glass bottomed 'boat' attached to camera and laser mount sub-frame. (b) System deployed in the Urie River, Scotland.

surface estimated to be approximately $5 \mathrm{~mm}$ thick (based on previous experience with similar structures and approximate estimates using conventional relationships). The 'boat' incorporates a trapezoidal-shaped water prism (e.g. Prasad 2000) to minimize both optical distortion caused by refraction and internal reflections that occur at the water-glass-air interfaces. The 'boat', cameras, and laser optics sit on a rigid subframe that allows the cameras and laser optics to be aligned, focused and calibrated in the laboratory prior to field deployment. In the field, the sub-frame is attached to a specially designed frame (bridge) and carriage assembly that allows the PIV system to be traversed in the streamwise $(0.5 \mathrm{~m})$ and transverse $(5.0 \mathrm{~m})$ directions. The bridge is constructed of aluminium extrusions (Kanya PVS), it spans $7.5 \mathrm{~m}$, weighs $150 \mathrm{~kg}$, and is designed so that at least one end of the bridge is anchored on the river bank. The other end of the bridge can be supported mid-river on stainless steel poles with tension straps tied to the far river bank to ensure stability. The turbulent wakes created by the bridge support elements are well clear of the measurement area (figure $1 b$ ). The laser, cameras and computer are powered by a portable $5 \mathrm{kVA}$ generator. Seeding (conifer pollen, $60-80 \mu \mathrm{m}$ diameter, $800-1000 \mathrm{~kg} \mathrm{~m}^{-3}$ density) is mixed with water at a concentration of $100 \mathrm{~g}^{-1}$ and injected into the river by a pump approximately $5 \mathrm{~m}$ upstream of the test section at a solids rate of $100 \mathrm{~g} \mathrm{~min}^{-1}$. The entire bridge, carriage, and laser and camera assembly can be installed at a field site by an eight person team in around $7 \mathrm{~h}$. Disassembly is faster (around $2 \mathrm{~h}$ ) leaving several hours for measurements during a single day deployment. The orientation of our coordinate system is shown in figure 1 . We will refer to the $x, y$ and $z$ axes and their associated velocity components $u, v$ and $w$ as the nominal streamwise, transverse and bed-normal (or vertical) directions and velocities, respectively. In practice, the laser light sheet was aligned visually to be parallel to the local mean flow direction by making use of the visible stream of tracer particles injected upstream.

A stereoscopic camera configuration was selected because it offers a number of benefits over a single orthogonal camera setup. Firstly, all three components of the velocity vector are resolved compared to just two components for a single camera configuration. The additional velocity component provides valuable information on the structure and dynamics of the flow field, particularly in the highly three-dimensional flow regions near the bed of open channels and in the wake of aquatic plants. Secondly, the stereoscopic configuration allows all cameras and laser optics to be placed above the river surface. This minimizes the disturbance to the flow field 
and limits camera vibration which can introduce additional error into the velocity measurements. The need to waterproof the camera and laser components is also removed. Thirdly, perspective errors which occur in single camera systems due to the unresolved out-of-plane velocity component (Raffel et al. 2007) are eliminated by the stereoscopic configuration. Finally, by taking advantage of the redundancy inherent in stereoscopic PIV, some velocity statistics can be calculated with a significantly reduced contribution of random measurement noise. In the following section, details of our implementation of the stereoscopic PIV method are outlined, including: calibration and stereoscopic reconstruction, cross-correlation algorithms, method of extracting the velocity of plant motion, and analysis of measurement errors.

\subsection{Stereoscopic PIV calibration}

Our stereoscopic PIV implementation is based on the 'mapping' method introduced by Willert (1997), where cross-correlation is performed on images that have been 'dewarped' to obtain a constant magnification across the image. The two-component vector fields from a pair of cameras are subsequently combined to reconstruct the three-component velocity field. Critical to both the image dewarping and velocity field reconstruction steps is a function which relates three-dimensional $(x, y, z$ : streamwise, transverse, bed-normal respectively) 'world' coordinates to corresponding two-dimensional image coordinates. To obtain it, we use a pinhole camera model (e.g. Calluaud \& David 2004) combined with a two-media refraction model (neglecting the contribution of the glass elements of the water prism) based on Maas (1996) and a misalignment correction based on Wieneke (2005). In total, 13 model parameters need to be estimated for each camera using a calibration procedure, including four intrinsic camera parameters $\left(f_{x}, f_{y}, i_{0}, j_{0}\right)$, six extrinsic camera parameters $\left(\alpha, \beta, \gamma, t_{x}\right.$, $\left.t_{y}, t_{z}\right)$, and three parameters for the refraction model $\left(\alpha_{g}, \beta_{g}, t_{z g}\right)$. Three additional parameters $\left(\alpha_{m}, \beta_{m}, t_{m}\right)$ apply to all cameras and are used to correct any misalignment between the laser light sheet and the calibration target. Here $f_{x}$ and $f_{y}$ are camera focal lengths, $i_{0}$ and $j_{0}$ are image origin coordinates, $\alpha, \beta, \gamma$ and $t_{x}, t_{y}, t_{z}$ are the three Euler rotation angles and three translations, respectively, that define the position and viewing direction of the camera. The refraction model parameters $\alpha_{g}, \beta_{g}$ and $t_{z g}$ are two rotation angles and one translation that give the position and orientation of the water-air interface, while misalignment parameters $\alpha_{m}, \beta_{m}$ and $t_{m}$ map the light sheet plane onto the calibration plane. Other parameters are available to incorporate lens distortions or to incorporate the refraction caused by the glass windows of the 'prism boat', but these parameters are not used in this study as they were found to not improve the calibration. The calibration procedure is carried out in a laboratory tank after final alignment and focusing of the cameras. The intrinsic, extrinsic, and refraction parameters are estimated for each camera based on a set of images of a two-sided calibration plate $(3 \mathrm{~mm}$ diameter dots spaced at $20 \mathrm{~mm}$ ) which is translated to different positions using a precision machined baseplate. The calibration images provide a set of point coordinates (the centres of each dot on the calibration plate image) and corresponding world coordinates (based on the known calibration plate geometry) allowing the model parameters to be optimized using an iterative leastsquare fit. Finally, the misalignment correction parameters are estimated from the experimental PIV images by ensemble cross-correlation between the dewarped images from the first and second cameras (Wieneke 2005). In this way, the precise position of the light sheet relative to the cameras does not need to be fixed in the laboratory and 


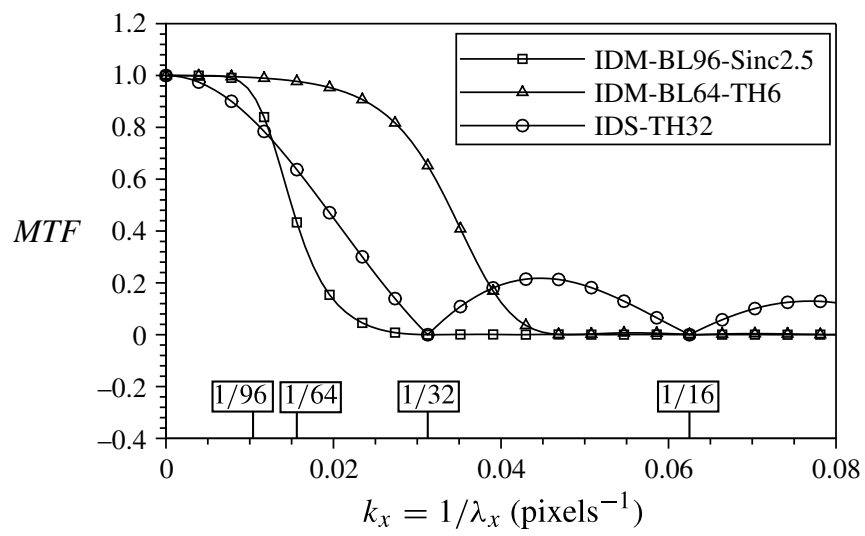

FIGURE 2. Comparison of transfer functions for different PIV algorithms: IDM-BL96Sinc2.5 is employed in this study; other algorithms are discussed in Cameron (2011).

some adjustment in the field is possible (as long as the light sheet remains within the camera depth of field).

\subsection{Cross-correlation algorithm}

A detailed description of our cross-correlation algorithm and evaluation of its performance is available in Cameron (2011). Some modifications were necessary to optimize for the field PIV images; these are described in this section.

Our PIV algorithm can be classed as an iterative deformation method (IDM) with windowed Fourier-transform-based cross-correlation. Two key features of the algorithm, which directly influence measurement noise, measurement resolution, number of outliers, and the number of iterations required to reach convergence are: (i) the size and weighting of the interrogation regions (image subsections used for cross-correlation analysis, e.g. Raffel et al. 2007); and (ii) the low-pass filtering of the velocity field after each iteration. To analyse the field PIV images, which have a scale factor of 12 pixels $\mathrm{mm}^{-1}$, we have selected Blackman-weighted $96 \times 96$ pixel $(8 \mathrm{~mm} \times 8 \mathrm{~mm})$ interrogation regions (BL96) with a 12 pixel $(1 \mathrm{~mm})$ grid spacing, and a low-pass filter based on a windowed sinc function (Sinc2.5). The modulation transfer function $(M T F)$ for this algorithm (IDM-BL96-Sinc2.5) has been estimated following Astarita (2007) and is given in figure 2. The MTF reflects the spatial averaging (low-pass filtering) of the velocity field associated with the cross-correlation algorithm. For an $M T F$ value of 0.9 , figure 2 indicates that the cutoff wavelength (resolution) for IDM-BL96-Sinc2.5 along the $k_{x}=1 / \lambda_{x}$ wavenumber axis is 92 pixels $(7.7 \mathrm{~mm})$, where $k_{x}$ is the wavenumber and $\lambda_{x}$ is the wavelength in the streamwise direction. This algorithm trades in some resolution relative to IDM-BL64-TH6 (Cameron 2011, figure 2) in return for improved robustness against outliers due to the larger interrogation regions. In comparison to the classic PIV method with 32 pixel unweighted interrogation regions (IDS-TH32, figure 2), IDMBL96-Sinc2.5 has slightly increased resolution, improved flatness in the pass band, efficient anti-aliasing due to steep roll-off and negligible side lobes, and significantly increased robustness due to having nine times more pixels in each interrogation region. Theoretical convergence for IDM-BL96-Sinc2.5 is eight iterations, defined here as the number of iterations required for the equivalent noise bandwidth $\left(E N B W_{q}\right)$ to reach $99.9 \%$ of its ultimate value. $E N B W_{q}$ is calculated for each iteration $(q)$ by integrating 
the squared transfer function predicted after each iteration:

$$
\operatorname{ENBW}_{q}=\int_{-\infty}^{\infty} \int_{-\infty}^{\infty}\left[\operatorname{MTF}_{q}\left(k_{x}, k_{z}\right)\right]^{2} \mathrm{~d} k_{x} \mathrm{~d} k_{z}
$$

where $M T F_{q}$ is the MTF estimated for $q$ iterations of the algorithm using the method of Astarita (2007), and $k_{z}$ is the wavenumber in the vertical direction. For comparison, theoretical convergence of IDM-BL64-TH6 is 36 iterations. There is additional filtering of the velocity field associated with the finite thickness of the light sheet $(\sim 1.5 \mathrm{~mm})$, but in this case the light sheet is quite thin and the effect is small relative to the filtering associated with the cross-correlation algorithm. The effect of the finite resolution of the measurement system is to reduce the contribution of high-wavenumber (small) eddies to the measured velocity variance. The magnitude of this effect depends on the flow field and cannot be easily quantified. For the present experiments, however, the cut-off wavelength of the measurements $(7.7 \mathrm{~mm})$ is small compared to the flow depth $(390 \mathrm{~mm})$ and therefore it is likely that the missing velocity variance is small.

A feature of the field PIV images is that some of the interrogation regions were intermittently occupied by plant material or void of sufficient seeding particles such that valid velocity vectors could not be obtained. In order to pre-empt these problems we introduce a measurement 'clipping' function, $\phi_{M}\left(x, z, t_{n}\right),\left(t_{n}\right.$ is the time step) defined as $\phi_{M}=1$ for valid interrogation regions (with sufficient seeding and absent of any plant), otherwise $\phi_{M}=0$. The mean value of $\phi_{M}\left(x, z, t_{n}\right)$ can be defined as the measurement porosity. The measurement clipping function serves two purposes: first it is passed to the cross-correlation algorithm so that bad interrogation regions can be handled appropriately by the algorithm, and second it is passed to velocity field post-processing routines so that velocity statistics are correctly calculated only over valid data. Regions where $\phi_{M}=0$ are identified in an image pre-processing stage using a type of signal to noise ratio. Each image is first decomposed into two parts: a plant image (by applying a median filter to the original image) and a seeding image (by subtracting the plant image from the original image). The 'signal' is then defined as the sum of the pixel intensities within an interrogation region for the seeding image, and the 'noise' is obtained as the sum of pixel intensities within an interrogation region for the plant image. For each interrogation region, if the signal divided by the noise is above a threshold value, $\phi_{M}$ is set to 1 , otherwise it is 0 . The threshold value was optimized by visual assessment and a trial and error approach on a subset of the PIV images. Once determined, it was applied globally throughout the image set. Within the PIV algorithm, regions of $\phi_{M}=0$ are replaced with interpolated or extrapolated velocity values from neighbouring valid data. This step is important with IDM PIV algorithms as it allows vector field low-pass filtering and interpolation to be performed in each iteration without inadvertently propagating bad vectors to adjacent interrogation regions. We emphasize that the interpolated/extrapolated values are only used within the cross-correlation algorithm. Time-averaged statistics of the velocity field are calculated incorporating only valid data as, for example, in the case of the first-order statistics:

$$
\bar{\theta}(x, z)=\frac{1}{\sum_{t_{n}=1}^{t_{n}=T}\left[\phi_{M}\left(x, z, t_{n}\right)\right]} \sum_{t_{n}=1}^{t_{n}=T}\left[\theta\left(x, z, t_{n}\right) \times \phi_{M}\left(x, z, t_{n}\right)\right]
$$


where $\theta$ is a flow variable, $t_{n}$ is the time step, and $T$ is the total number of time steps. The $\phi_{M}$ parameter is also used in calculating correlation functions and spectra; equations are given where appropriate in the following sections. Potential measurement uncertainties associated with flow regions having small values of measurement porosity are limited by only presenting data for which $\overline{\phi_{M}}>0.75$.

\subsection{Calculating plant velocity}

The field PIV images contained enough detail of the fluctuating plant to extract estimates of the vertical $w_{p}(x)$ and transverse $v_{p}(x)$ plant velocity components. We selected rectangular interrogation regions $(96 \times 1024$ pixels, $8 \mathrm{~mm} \times 85 \mathrm{~mm})$ which were sufficiently high to cover the entire visible plant cross-section, but narrow enough that plant velocities could be measured as a function of streamwise position. Standard cross-correlation applied to median filtered PIV images (to remove seeding particles) resulted in a rather wide peak in the correlation function (proportional to the width of the plant) and therefore poor accuracy in estimating the displacement. To improve cross-correlation performance, we have employed Wernet's (2005) symmetric phase only filtering (SPOF) which makes the correlation function more sensitive to the highwavenumber content of the image (i.e. the sharply defined edges of the plant stems and leaves). The SPOF takes the form of a weighting function $C\left(k_{m}, k_{n}\right)$ applied to the cross-spectral density $G\left(k_{m}, k_{n}\right) H^{*}\left(k_{m}, k_{n}\right)$ between a pair of interrogation regions:

$$
C\left(k_{m}, k_{n}\right)=\frac{1}{\sqrt{\left|G\left(k_{m}, k_{n}\right)\right|} \sqrt{\left|H\left(k_{m}, k_{n}\right)\right|}},
$$

where $G$ and $H$ are the Fourier transforms of the first and second interrogation regions, $H^{*}$ is the complex conjugate of $H$, and $k_{m}$ and $k_{n}$ are wavenumbers in the $m$ and $n$ dewarped image directions. The cross-correlation function $\varphi$ is then calculated as the inverse Fourier transform $\left(\mathrm{FFT}^{-1}\right)$ of the weighted cross-spectral density, which for $C=1$ is the standard Fourier transform based cross-correlation:

$$
\varphi=\mathrm{FFT}^{-1}\left[C\left(k_{m}, k_{n}\right) G\left(k_{m}, k_{n}\right) H^{*}\left(k_{m}, k_{n}\right)\right] .
$$

The displacement of the correlation peak is estimated only in the vertical $(n)$ image direction which is sensitive to vertical and transverse displacements of the plant. By combining the displacements estimated from a pair of stereoscopic cameras, the vertical $w_{p}(x)$ and transverse $v_{p}(x)$ plant velocity components are recovered. Due to the extended interrogation regions, the measured velocities approximate the cross-sectional average of plant velocity fluctuations.

\subsection{Stereoscopic velocity field reconstruction}

Two-component velocity fields estimated using cross-correlation on dewarped images are combined from two cameras to reconstruct the three-component velocity field according to Raffel et al. (2007) as:

$$
\left[\begin{array}{lll}
1 & 0 & \psi_{1 m} \\
0 & 1 & \psi_{1 n} \\
1 & 0 & \psi_{2 m} \\
0 & 1 & \psi_{2 n}
\end{array}\right] \quad\left[\begin{array}{c}
\Delta_{x} \\
\Delta_{z} \\
\Delta_{y}
\end{array}\right]=\left[\begin{array}{c}
\Delta_{1 m} \\
\Delta_{1 n} \\
\Delta_{2 m} \\
\Delta_{2 n}
\end{array}\right]
$$

where $\Delta_{c m}$ and $\Delta_{c n}$ are the two displacement components estimated from images from the $c$ camera $(c=1,2), \Delta_{x}, \Delta_{y}$ and $\Delta_{z}$ (pixels) are the three displacement components in the $x, y, z$ directions (figure $1 a$ ) and $\psi_{c m}$ and $\psi_{c n}$ are calibration factors calculated 
at the centre of each interrogation region using the camera calibration model. The $\psi_{\mathrm{cm}}$ and $\psi_{c n}$ values indicate the shift in dewarped image coordinate (respectively in the $m$ and $n$ directions) corresponding to a unit displacement in the $y$ (out-of-plane) direction and are equivalent to the tangents of the local view angles.

Equation (2.5) is an overdetermined system of linear equations (four equations, three unknowns). It can be solved using a least-squares method (Raffel et al. 2007) or by calculating exact solutions to subsets of the four equations (e.g. Prasad 2000). In the latter case, a redundant estimate for one of the velocity components may be obtained and it is standard practice to average together the redundant estimates to reduce the variance of the measurement noise in that component by a factor of two (Prasad 2000). More efficient use of the redundancy in (2.5) can be made by storing the redundant estimates separately rather than averaging them together. Following the method introduced for acoustic Doppler velocimeters by Hurther \& Lemmin (2001), some velocity statistics can then be calculated which have significantly reduced noise contribution. This approach has not previously been tested with stereoscopic PIV data and so a brief evaluation is given in the following section. For the present camera configuration, the redundancy inherent in (2.5) falls substantially on the $\Delta_{x}$ displacement component, although in the general case, it may be shared between all of the displacement components. Based on (2.5), we can write:

$$
\begin{aligned}
& v=\Delta_{y}\left(M \Delta_{l s}\right)^{-1}=\left(\Delta_{1 n}-\Delta_{2 n}\right)\left(\psi_{1 n}-\psi_{2 n}\right)^{-1}\left(M \Delta_{l s}\right)^{-1}, \\
& w=\Delta_{z}\left(M \Delta_{l s}\right)^{-1}=\left(\Delta_{1 n}\right)\left(M \Delta_{l s}\right)^{-1}-\psi_{1 n} v=\left(\Delta_{2 n}\right)\left(M \Delta_{l s}\right)^{-1}-\psi_{2 n} v \text {, } \\
& u_{[1]}=\left(\Delta_{1 m}\right)\left(M \Delta_{l s}\right)^{-1}-\psi_{1 m} v \text {, } \\
& u_{[2]}=\left(\Delta_{2 m}\right)\left(M \Delta_{l s}\right)^{-1}-\psi_{2 m} v \text {, } \\
& u=0.5\left(u_{[1]}+u_{[2]}\right) \text {, }
\end{aligned}
$$

where $M$ is a scale factor of the dewarped images (pixels $\mathrm{mm}^{-1}$ ), $\Delta_{l s}(\mathrm{~ms})$ is the time separation between laser pulses, $u_{[1]}$ and $u_{[2]}$ are redundant estimates of the $u$ velocity component, and $u, v$, and $w$ are the velocity components $\left(\mathrm{m} \mathrm{s}^{-1}\right)$ in the $x, y$, and $z$ directions respectively.

\subsection{Noise reduction}

The redundancy in the streamwise velocity measurement can be used to calculate velocity variance with a substantially reduced contribution of measurement noise. The instantaneous measured velocity fluctuation $\left(u^{\prime}=u-\bar{u}\right)$ can be decomposed into the sum of the actual velocity fluctuation $\left(u_{a}{ }^{\prime}\right)$ and the measurement error $\left(\varepsilon_{u}{ }^{\prime}\right)$ as $u^{\prime}=u_{a}{ }^{\prime}+\varepsilon_{u}{ }^{\prime}$. The measured velocity variance can then be written:

$$
\overline{u^{\prime} u^{\prime}}=\overline{\left(u_{a}{ }^{\prime}+\varepsilon_{u}{ }^{\prime}\right)\left(u_{a}{ }^{\prime}+\varepsilon_{u}{ }^{\prime}\right)}=\overline{u_{a}{ }^{\prime} u_{a}{ }^{\prime}}+\overline{\varepsilon_{u}{ }^{\prime} \varepsilon_{u}^{\prime}}+2 \overline{u_{a}{ }^{\prime} \varepsilon_{u}{ }^{\prime}},
$$

where the term $2 \overline{u_{a}{ }^{\prime} \varepsilon_{u}{ }^{\prime}}$ vanishes if the measurement error is not correlated with the actual velocity fluctuation. The measured velocity variance therefore includes contributions from the actual velocity variance and the variance of the random measurement error. If redundant estimates of the velocity fluctuation $\left(u_{[1]}{ }^{\prime}, u_{[2]}{ }^{\prime}\right)$ are available, (2.11) can be rewritten as:

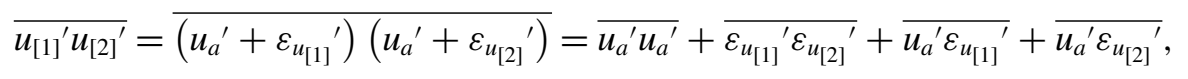

where $\varepsilon_{u_{[1]}}{ }^{\prime}$ and $\varepsilon_{u_{[2]}}{ }^{\prime}$ are the measurement errors associated with $u_{[1]}{ }^{\prime}$ and $u_{[2]}{ }^{\prime}$ respectively. The third and fourth terms on the right vanish if the measurement error is

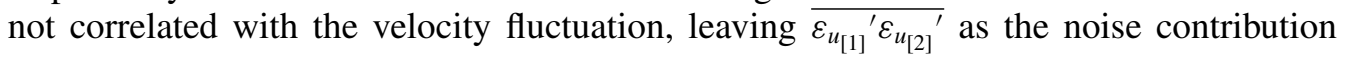




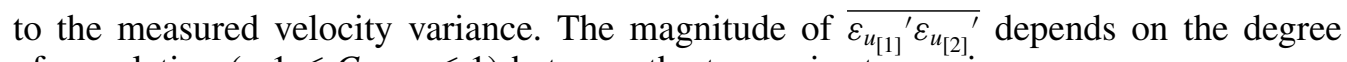
of correlation $\left(-1 \leqslant C_{\varepsilon_{u_{[12]}}} \leqslant 1\right)$ between the two noise terms, i.e.

$$
\overline{\varepsilon_{u_{[1]}{ }^{\prime}} \varepsilon_{u_{[2]}}{ }^{\prime}}=C_{\varepsilon_{u_{[12]}}} \sqrt{\left(\overline{{\varepsilon_{u_{[1]}}{ }^{\prime} \varepsilon_{u_{[1]}}}^{\prime}}\right)\left(\overline{\varepsilon_{u_{[2]}{ }^{\prime} \varepsilon_{u_{[2]}}}}\right) .}
$$

In the ideal case $C_{\varepsilon_{u[12]}}$ approaches zero, and if additionally the measurement error is

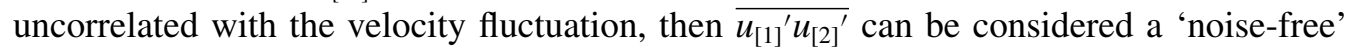
estimate of the velocity variance. In practice, although $u_{[1]}$ and $u_{[2]}$ are measured by different cameras, some correlation between the noise terms might be expected as the same particles are imaged by both cameras, albeit from different angles. Furthermore the equations for $u_{[1]}$ and $u_{[2]}$ (2.8) and (2.9) both include the transverse velocity $v$. In the present study, however, the multiplying factors $\psi_{1 m}$ and $\psi_{2 m}$ are quite small, increasing from zero at the centre of the image to around $\left|\psi_{c m}\right|=0.2$ at the left and right edges. Nevertheless, part of the measurement error in $v$ will contribute to $\overline{u_{[1]} u_{[2]}}$.

For the camera configuration used in our field experiments, this approach is limited to reducing the noise in statistics of the streamwise velocity component. The noise level in the other components can, however, still be estimated. Based on (2.5), by assuming $\psi_{1 n}=-\psi_{2 n}$ (for a symmetric camera system) and $\psi_{1 m}=\psi_{2 m}=0$, and applying standard equations for error propagation, it can be shown that

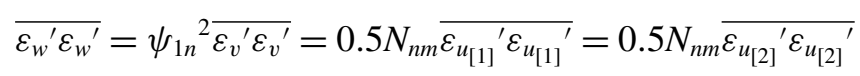

and

$$
\overline{\varepsilon_{v}{ }^{\prime} \varepsilon_{w}{ }^{\prime}}=\overline{\varepsilon_{u}{ }^{\prime} \varepsilon_{w}{ }^{\prime}}=\overline{\varepsilon_{u}{ }^{\prime} \varepsilon_{v}{ }^{\prime}} \sim 0,
$$

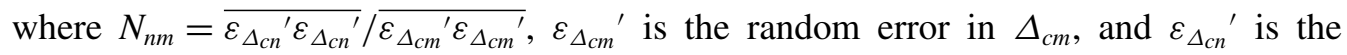
random error in $\Delta_{c n}$, and it is assumed that the error variance is the same for each

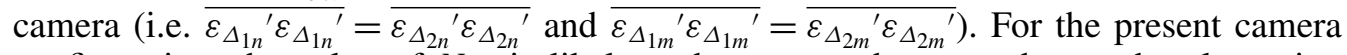
configuration, the value of $N_{n m}$ is likely to be greater than one due to the elongation of particle images induced by the image dewarping process. Its value can be estimated with the help of computer-generated PIV images.

Artificial PIV images were generated using a procedure described in Cameron (2011), but extended here to generate a stereoscopic pair of images by applying the camera calibration model to transform simulated three-dimensional particle coordinates to image coordinates for a pair of cameras. The simulated cameras were positioned similarly to the real cameras used in the field experiments $\left(63^{\circ}\right.$ viewing angle). Other parameters of the simulation were: seeding concentration of $9 \times 10^{-3}$ particles per pixel, particle image diameter of 2.1 pixels, background intensity of 6 grey levels ( 8 bit quantization), random additive noise with standard deviation 1.4 grey levels, maximum particle brightness of 500 grey levels (reflecting some saturation of the 8 bit image), and fill factor of 0.6. These parameters were selected to approximate the experimental PIV images obtained in the field. A series of $256 \times 256$ pixel images were generated, each with a uniform displacement field across the image, but with the displacement systematically varied over a set of $4 \times 10^{5}$ images to uniformly cover the range $0<\Delta_{c m}<2$ and $0<\Delta_{c n}<4.4$ pixels which corresponds to two full cycles of the peak locking error (Raffel et al. 2007) in each direction. Note that the peak locking error typically has a period of 1 pixel, but when images are dewarped, by in this case stretching the image by a factor of 2.2 in the $n$ direction, the peak locking period is stretched by the same factor. The simulated images were analysed using the 
same algorithm as was used for the field experiment images, and the error variance for each component was obtained as:

$$
\overline{\varepsilon_{u_{[1]}{ }^{\prime} \varepsilon_{u_{[1]}}{ }^{\prime}}}=\overline{\varepsilon_{u_{[2]}{ }^{\prime} \varepsilon_{u_{[2]}{ }^{\prime}}}}=1.06 \overline{\varepsilon_{v}{ }^{\prime} \varepsilon_{v}{ }^{\prime}}=0.27 \overline{\varepsilon_{w}{ }^{\prime} \varepsilon_{w}{ }^{\prime}},
$$

indicating a value of $N_{n m}=7.4$. This relationship is used in $\S 3.1$ to estimate the variance of the errors in the vertical and transverse velocity components. From the simulation, the correlation coefficient between the errors in $u_{[1]}$ and $u_{[2]}$ was found to be very small $\left(C_{\varepsilon_{u_{[12]}}}=1.4 \times 10^{-3}\right)$. The simulation data also indicate that the ratio $\overline{u_{a}{ }^{\prime} \varepsilon_{u_{[1]}{ }^{\prime}}} / \overline{u_{a}{ }^{\prime} u_{a}^{\prime}} \approx \overline{u_{a}{ }^{\prime} \varepsilon_{u_{[2]}}} / \overline{u_{a}{ }^{\prime} u_{a}{ }^{\prime}}$ is of the order $10^{-6}$ for the present experiments, confirming that the third and fourth terms on the right-hand side of (2.12) can be safely neglected. It is therefore reasonable to assume that $\overline{u_{[1]} u_{[2]}^{\prime}}$ has significantly

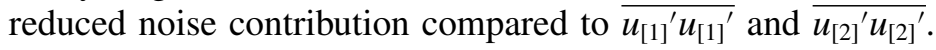

Higher-order statistics can also be estimated using redundant velocity estimates to reduce noise contribution. For example, by calculating the velocity skewness $(S)$ and kurtosis $(K)$ as:

$$
\begin{gathered}
S_{u}=\frac{\overline{u^{\prime} u^{\prime} u^{\prime}}}{\left(\overline{u_{[1]^{\prime} u_{[2]^{\prime}}}}\right)^{3 / 2}}, \\
K_{u}=\frac{\overline{u_{[1]^{\prime} u_{[1]^{\prime}} u_{[2]} u_{[2]}}}+\left(\overline{u_{[1]^{\prime} u_{[2]}}}\right)\left(\overline{u_{[1]^{\prime}} u_{[2]}}\right)-\left(\overline{u_{[1]^{\prime}} u_{[1]^{\prime}}}\right)\left(\overline{u_{[2]} u_{[2]}}\right)}{\left(\overline{u_{[1]^{\prime} u_{[2]}}}\right)^{2}}-3 .
\end{gathered}
$$

The measurement noise is eliminated if it is uncorrelated with the velocity fluctuation and if the noise correlation $C_{\varepsilon_{u_{[12]}}}$ is zero. Again, (2.17) and (2.18) can only be applied for the streamwise velocity component for the present camera configuration. The noise contribution to the measured skewness and kurtosis for other velocity components can be estimated assuming that the random errors have a Gaussian distribution, using

$$
\begin{aligned}
& S_{v a}=\frac{\overline{v_{a}{ }^{\prime} v_{a}{ }^{\prime} v_{a}{ }^{\prime}}}{\overline{v_{a}{ }^{\prime} v_{a}{ }^{3 / 2}}}=\left(1+N_{v}\right)^{3 / 2} S_{v}, \\
& S_{w a}=\frac{\overline{w_{a}{ }^{\prime} w_{a}{ }^{\prime} w_{a}{ }^{\prime}}}{\overline{w_{a}{ }^{\prime} w^{\prime}}}=\left(1+N_{w}\right)^{3 / 2} S_{w}, \\
& K_{v a}=\frac{\overline{v_{a}^{\prime} v_{a}{ }^{\prime} v_{a}{ }^{\prime} v_{a}{ }^{\prime}}}{{\overline{v_{a}{ }^{\prime} v_{a}{ }^{2}}}^{2}}-3=\left(1+N_{v}\right)^{2} K_{v}, \\
& K_{w a}=\frac{\overline{w_{a}{ }^{\prime} w_{a}{ }^{\prime} w_{a}{ }^{\prime} w_{a}{ }^{\prime}}}{\overline{w_{a}{ }^{\prime} w_{a}{ }^{2}}}-3=\left(1+N_{w}\right)^{2} K_{w},
\end{aligned}
$$

where $S_{v}$ and $S_{w}$ are the measured transverse and vertical velocity skewness, $K_{v}$ and $K_{w}$ are the measured transverse and vertical velocity kurtosis. Actual (or noise-free) velocity fluctuations $\left(v_{a}{ }^{\prime}, w_{a}{ }^{\prime}\right)$, skewness $\left(S_{v a}, S_{w a}\right)$ and kurtosis $\left(K_{v a}, K_{w a}\right)$ are denoted with the subscript ' $a$ '. The terms $N_{v}$ and $N_{w}$ are the noise to signal ratios defined as:

$$
N_{v}=\frac{\overline{\varepsilon_{v}^{\prime} \varepsilon_{v}{ }^{\prime}}}{\overline{v_{a}^{\prime} v_{a}^{\prime}}} \quad \text { and } \quad N_{w}=\frac{\overline{\varepsilon_{w}^{\prime} \varepsilon_{w}^{\prime}}}{\overline{w_{a}^{\prime} w_{a}^{\prime}}}
$$

which can be estimated from (2.16) and the experimentally measured velocity variance. These relationships are used in $\S 4.3$ to estimate noise contributions to measured velocity skewness and kurtosis. 
(a)

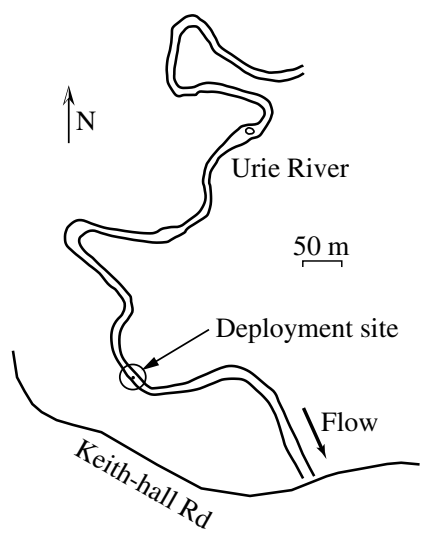

(b)

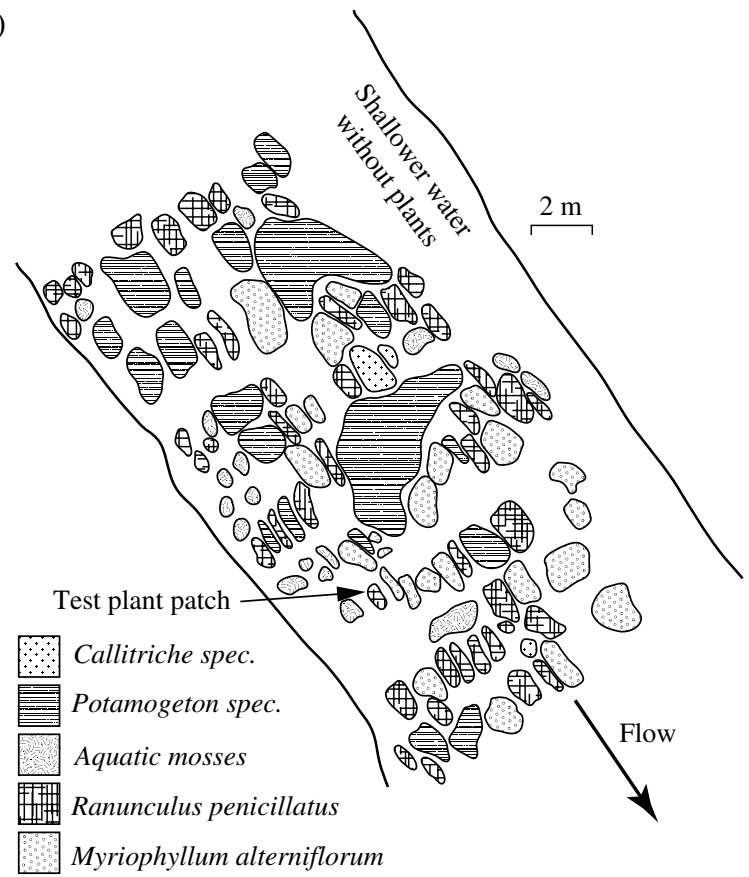

FIGURE 3. (a) Sketch of the field deployment site. (b) Aquatic plant species near the test section of the Urie River.

\section{Field site and experiments}

The site selected for the field deployment was on the Urie River, near the town of Inverurie and $26 \mathrm{~km}$ from Aberdeen City. An approximately straight section of the river was identified (figure $3 a$ ) with convenient vehicle access and a rich abundance of aquatic plants, including species from the Myriophyllum, Ranunculus, Potamogeton, and Callitriche genera, along with various aquatic mosses (figure $3 b$ ). The gravel bed at this river reach had a median particle size of $35 \mathrm{~mm}$ (estimated from a random sample of 117 particles) and featured intermittent sandy patches and occasional large boulders. At the test section the river was $12.9 \mathrm{~m}$ wide (figure 4), the average flow depth was $0.39 \mathrm{~m}$, the flow rate was $2.7 \mathrm{~m}^{3} \mathrm{~s}^{-1}$, and the water surface slope was $1.5 \pm 0.4 \times 10^{-3}$. The Reynolds number based on flow depth and mean velocity was $1.52 \times 10^{5}$ and the Froude number was 0.28. Assessment of velocity time series (not shown) and observations of the river water surface elevation throughout the deployment suggest that the flow conditions were steady.

A set of PIV measurements were made of the flow field around a Ranunculus penicillatus plant patch located $2.8 \mathrm{~m}$ from the right river bank. The maximum dimensions of the patch were approximately $400 \mathrm{~mm}$ long, $200 \mathrm{~mm}$ wide, and $100 \mathrm{~mm}$ high. This particular patch was selected because of its size in relation to the PIV field of view and its isolation from other plants and large boulders. Threeminute PIV recordings were made at three measurement locations, starting near the free end of the plant patch and subsequently incremented by $130 \mathrm{~mm}$ in the downstream direction. The total measurement coverage was a planar region $400 \mathrm{~mm}$ in the streamwise direction and $320 \mathrm{~mm}$ in the vertical direction and aligned with the centreline of the plant (figure $5 a$ ). The recording rate was 30 image pairs per second, 


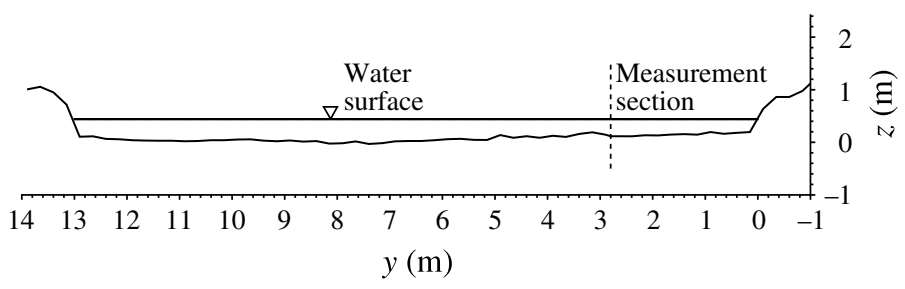

FIGURE 4. Cross-section of the Urie River near the test section.

(a)

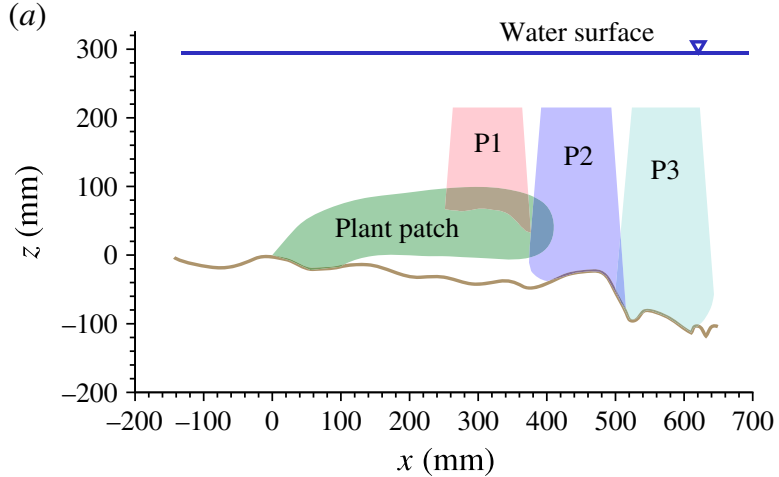

(b)

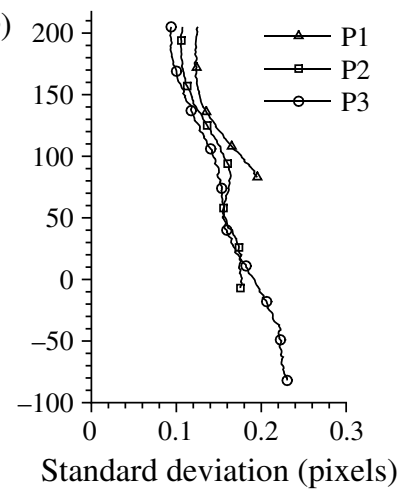

FIGURE 5. (Colour online) (a) PIV measurement coverage (P1, P2, P3) relative to the plant patch and local bed topography. (b) Standard deviation of measurement noise for the three measurement positions.

but due to a technical issue, some of the frames were later found to not be viable, resulting in an average of 20 image pairs per second. Missing time steps are assigned $\phi_{M}=0$ allowing statistical quantities to be estimated using only valid data.

\subsection{Measurement noise}

Based on the redundant estimates of the streamwise velocity component, the variance of the noise can be estimated as:

$$
0.5\left(\overline{\varepsilon_{u_{[1]}{ }^{\prime} \varepsilon_{u_{[1]}}}}+\overline{\varepsilon_{u_{[2]}{ }^{\prime} \varepsilon_{u_{[2]}}{ }^{\prime}}}\right)=0.5\left(\overline{u_{[1]}^{\prime} u_{[1]}{ }^{\prime}}+\overline{u_{[2]} u_{[2]}^{\prime}}-2 \overline{u_{[1]} u_{[2]}}\right) .
$$

This noise term is plotted in the form $\sqrt{0.5} M \Delta_{l s}\left(\overline{\varepsilon_{u_{[1]}{ }^{\prime} \varepsilon_{u_{[1]}}}}+\overline{\varepsilon_{u_{[2]}{ }^{\prime} \varepsilon_{u_{[2]}}}}\right)^{0.5}$ in figure $5(b)$, which is the standard deviation of the error in displacement units (pixels), allowing comparison with previous studies of PIV error (here $M=12$ pixels $\mathrm{mm}^{-1}$ and $\left.\Delta_{l s}=1 \mathrm{~ms}\right)$. Figure $5(b)$ indicates that the standard deviation of the measurement noise in the streamwise displacement component is approximately the same for each of the three measurement positions and increases from around 0.1 pixels for large $z$ (near the free surface) to around 0.2 pixels near the bed. The increase in error approaching the bed reflects the varying magnification of the source images and deteriorating image conditions with distance from the cameras due to light sheet intensity fall-off. The magnitude of the error is comparable to that obtained from computer simulations when considering a significant out-of-plane displacement component (e.g. Nobach \& Bodenschatz 2009; Cameron 2011). Values of the noise to 
signal ratio terms (2.23) for the transverse and vertical velocity components can be estimated as $N_{v}=0.02$ and $N_{w}=0.07$ in the wake of the plant patch.

\section{Flow turbulence and plant fluctuations}

\subsection{Mean velocity field}

Mean velocity streamlines combined for the three measurement planes (figure 6) indicate that the flow does not separate from the plant patch and no recirculation zone forms. Folkard (2011a) defines this as the 'canopy through-flow' regime, but the flow and patch conditions for its existence are yet to be identified for real plants. In contrast, the small rock immediately behind the plant patch shows clear signs of separation and recirculation, highlighting the potentially different mechanisms of drag for these two objects. Bluff bodies, such as the rock behind the plant patch, produce drag mainly through the differential pressure between their upstream and downstream surfaces which occurs due to flow separation. Drag on aquatic plants, however, due to their flexibility, porosity, and large wetted surface area, may be dominated by viscous drag (Nikora \& Nikora 2007) which forms due to the velocity gradient at the plant surfaces. Although figure 6 is consistent with the proposed conjecture, this hypothesis is difficult to test experimentally as flow separation and pressure drag may occur at several different plant scales (plant patch, individual plant, stem, leaf). Recent experimental studies have measured drag forces at each of these scales (e.g. Albayrak et al. 2012; Nikora et al. 2012; Siniscalchi \& Nikora 2012; Siniscalchi et al. 2012; Siniscalchi \& Nikora 2013); however, separating viscous/pressure drag contributions directly still exceeds experimental capability. Further complicating the viscous/pressure drag argument is that simple scaling relationships such as $F_{D} \propto u^{2}$ for pressure drag and $F_{D} \propto u^{1}$ for viscous drag (where $F_{D}$ is drag force on the plant) cannot easily be applied to aquatic plants as they have the tendency to change their structure in response to the velocity field. This so-called 'reconfiguration' (Vogel 1994; de Langre 2008) can change the wetted surface area, the effective frontal area and the drag coefficient (through streamlining) of the plant as a function of flow velocity, thereby complicating interpretation of force scaling with flow velocity.

The plant drag, whether viscous or pressure dominated, is a sink of momentum and introduces a free shear layer (and associated inflection in the $\bar{u}(z)$ profile) at the interface between the retarded flow in the wake of the plant and the background channel flow (figure 7). The inflectional form of the mean velocity profile is suggested to lead to the Monami phenomenon in aquatic plant canopies (Ghisalberti \& Nepf 2002; Nezu \& Sanjou 2008; Nepf 2012) and dominate local turbulence characteristics due to a periodic production of vortices (Kelvin-Helmholtz instability). For a single isolated plant patch, however, the mean flow in the wake is distinctly three-dimensional and exposed to high background turbulence levels which would tend to disrupt any periodic vortex formation mechanisms. The shear layer may nevertheless be associated with high levels of turbulence production; the distribution of the Reynolds stresses and their budget terms are examined in the following section. Potential periodicity of the velocity in the plant wake is examined in $\S 4.5$. Figure 7(b) illustrates the streamwise momentum recovery in the wake of the plant for the $x$ coordinates marked by circles in figure $7(a)$. The streamwise velocity in the wake is steadily increasing with increasing $x$, and the corresponding decay of the maximum velocity gradient is apparent. 


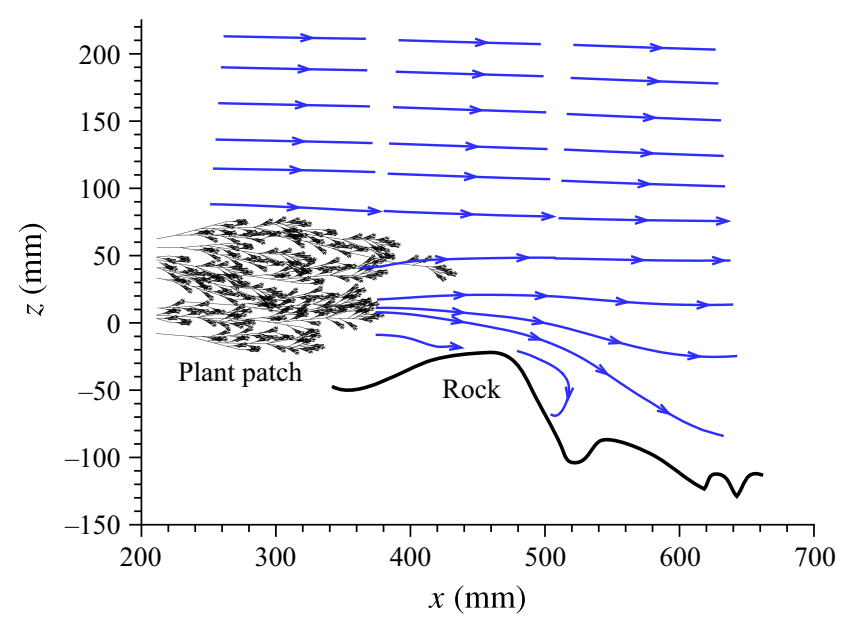

FIGURE 6. (Colour online) Velocity streamlines in the flow region around a Ranunculus plant patch.
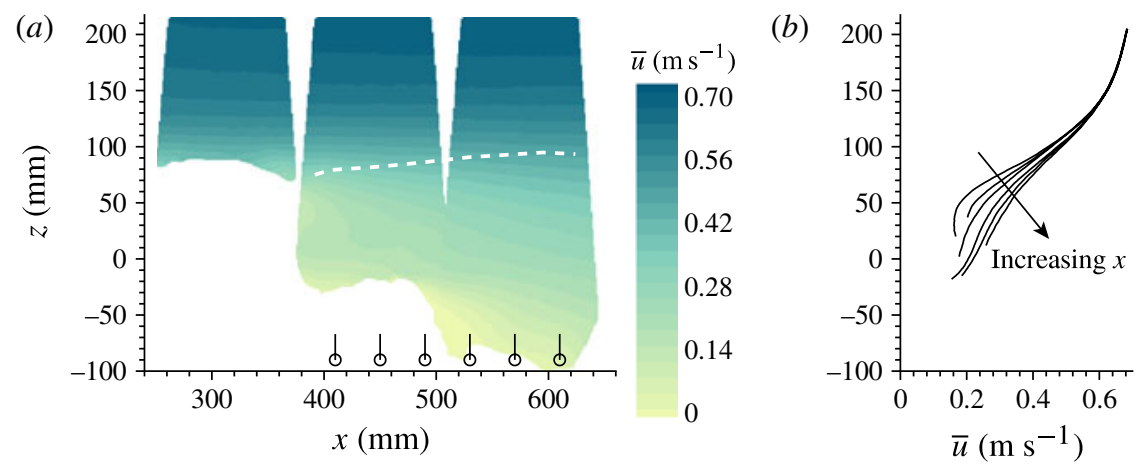

FIgURE 7. (Colour online) Time-averaged streamwise velocity distribution: $(a)$ around a Ranunculus plant patch, and $(b)$ in the wake of the plant patch for $x$ coordinates corresponding to circle symbols in $(a)$. Dashed line indicates local maximum in the $\partial \bar{u} / \partial z$ distribution.

\subsection{Reynolds stresses and their budget terms}

The normal Reynolds stresses $\left(\overline{u^{\prime} u^{\prime}}, \overline{v^{\prime} v^{\prime}}, \overline{w^{\prime} w^{\prime}}\right)$ and the primary Reynolds shear stress $\left(-\overline{u^{\prime} w^{\prime}}\right)$ all attain maximum values near the shear layer in the wake of the plant (figure 8). In general, the distribution of Reynolds stresses in the patch wake may depend on a variety of patch and approach flow conditions such as patch length and width, the distribution and shape of plant stems and leaves within the patch, the flexibility of the plants, the approach flow Reynolds number, and the flow depth to patch height ratio. For example, in contrast to our study, the peak Reynolds stress for Folkard's (2005) model seagrass canopy formed several patch heights downstream of the patch and near the reattachment point of the separated flow. It is not yet 

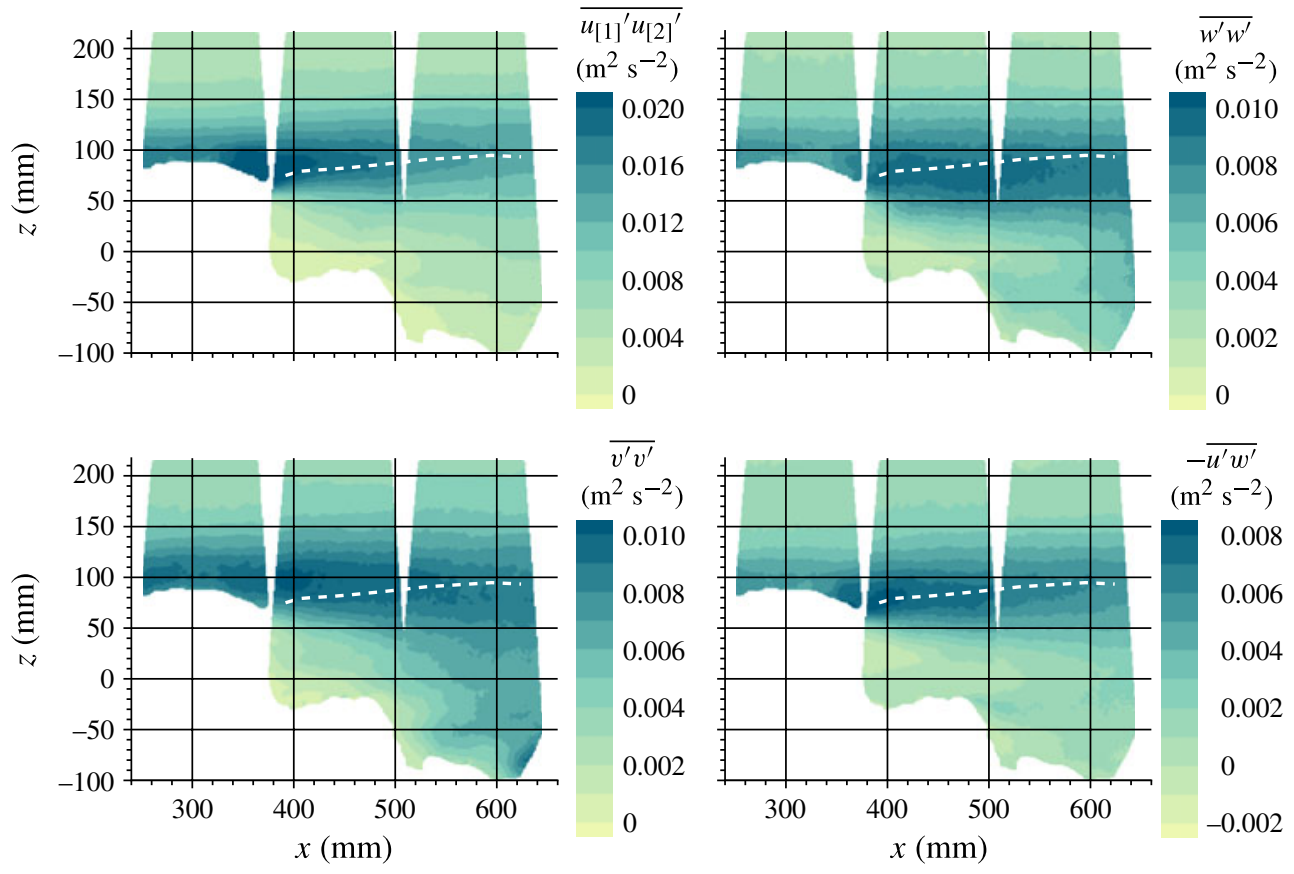

FIGURE 8. (Colour online) Reynolds normal stresses and the primary Reynolds shear stress. Dashed lines indicate local maximum in the $\partial \bar{u} / \partial z$ distribution.

clear if natural patches of Ranunculus penicillatus form similar wake features under different flow and patch conditions. Secondary Reynolds shear stresses (not shown) were found to be an order of magnitude smaller than the primary Reynolds shear stress as might be expected (due to symmetry) near the centreline of the plant. The Reynolds stress correlation coefficient $-\overline{u^{\prime} w^{\prime}} /\left(\overline{u^{\prime} u^{\prime}} \overline{w^{\prime} w^{\prime}}\right)^{0.5}$, which reflects the efficiency of the turbulent fluctuations in redistributing momentum, has a maximum value of 0.61 in the plant wake $(x=400, z=75)$, slightly larger than the 0.4-0.5 typical for open channel flows (Nezu \& Nakagawa 1993), the 0.5 found for terrestrial canopies (Raupach, Finnigan \& Brunet 1996), and the 0.5 found in the wake of a cylinder (Cantwell \& Coles 1983).

The transverse and vertical normal stresses have similar magnitudes to each other in the plant wake $\left(\overline{v^{\prime} v^{\prime}} / \overline{w^{\prime} w^{\prime}}=1-1.2\right)$ which is smaller than the ratio 1.65 typical for open channel flows (Nezu \& Nakagawa 1993), but closer to the ratio of 1.2 measured for a plane mixing layer by Wygnanski \& Fiedler (1970). The streamwise normal stress $\overline{u^{\prime} u^{\prime}}$ is found to decay with increasing $x$ much faster than the other components. In the far wake $(x=600 \mathrm{~mm}) \overline{u^{\prime} u^{\prime}}$ has reduced to $71 \%$ of its near wake $(x=400 \mathrm{~mm})$ maximum. Corresponding values for $\overline{v^{\prime} v^{\prime}}$ and $\overline{w^{\prime} w^{\prime}}$ are $91 \%$ and $85 \%$ respectively. Subtle differences in the elevations where the maximum variance occurs can be seen between the different components of the Reynolds stress tensor. Local maxima in both $\overline{u^{\prime} u^{\prime}}(z)$ and $\overline{u^{\prime} w^{\prime}}(z)$ tend to higher elevations with increasing $x$ following the mean shear layer and reflecting the expansion of the wake region into the outer flow. The trend for $\overline{w^{\prime} w^{\prime}}(z)$ is nearly horizontal, and for $\overline{v^{\prime} v^{\prime}}(z)$ it is downward. The reason for these different trends is not clear, but further understanding might be gained by 
considering the budget equation for the Reynolds stresses:

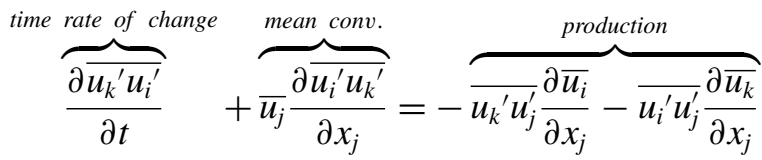

$$
\begin{aligned}
& -\overbrace{\frac{1}{\rho} \frac{\partial \overline{u_{k}^{\prime} p^{\prime}}}{\partial x_{i}}-\frac{1}{\rho} \frac{\partial \overline{u_{i}^{\prime} p^{\prime}}}{\partial x_{k}}}^{\text {pres. trans. }}-\overbrace{\frac{\partial \overline{u_{k}^{\prime} u_{j}^{\prime} u_{i}^{\prime}}}{\partial x_{j}}}^{\text {turb. trans. }}+\overbrace{v \frac{\partial}{\partial x_{j}}\left(\frac{\partial \overline{u_{i}^{\prime} u_{k}^{\prime}}}{\partial x_{j}}\right)}^{\text {visc. trans. }}
\end{aligned}
$$

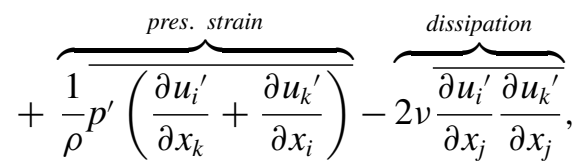

where $\rho$ is fluid density, $v$ is kinematic fluid viscosity, and $p$ is fluid pressure. The free indices $\left(i\right.$ and $k$ ) can take the values 1,2 or 3 where $u_{1}, u_{2}, u_{3}$ correspond to the velocity components $u, v$ and $w$ and $x_{1}, x_{2}, x_{3}$, correspond to the $x, y$, and $z$ directions respectively (figure $1 a$ ). The dummy index $j$ implies summation over all possible values of $j(j=1,2,3)$ in accordance with the Einstein summation convention. Overbars indicate time- (ensemble-) averaged values and the prime symbol defines the deviation of an instantaneous variable from its time-averaged value (e.g. $\left.u^{\prime}=u-\bar{u}\right)$.

The Reynolds stress budget equation can be derived from the Navier-Stokes (NS) momentum conservation equation in three steps. First derive an equation for the fluctuating velocity by subtracting the time average of the NS equation from the NS equation. Second, multiply the equation for the velocity fluctuation by $u_{k}$ and time average the resulting equation. Third, exchange the free indices $(i$ and $k)$ in the equation developed in step 2 and add this new equation to the original equation in step 2 to give (4.1). It can be noted that the budget equation for turbulent kinetic energy is obtained by taking half the trace of (4.1). Equation (4.1) has received considerable attention as a framework to develop closure models for the Reynolds-averaged NS equations. Distribution of the terms in (4.1) can also provide some insight into the turbulence in the wake of the plant patch, and in the present study this is our primary interest.

In a uniform, two-dimensional channel flow $\bar{v}=\bar{w}=\partial / \partial x=\partial / \partial y=0$ and the only non-zero normal stress production term is in the $\overline{u^{\prime} u^{\prime}}$ budget. Variance is redistributed from $\overline{u^{\prime} u^{\prime}}$ to the other normal stress components by the pressure-strain correlation term which is traceless and therefore does not appear in the total turbulent kinetic energy balance. Away from boundaries, the dissipation rate is expected to be approximately equal in each of the normal stress budgets due to local isotropy, if the Reynolds number is reasonably high (Davidson 2004). Dissipation in the $\overline{u^{\prime} w^{\prime}}$ budget is typically small, and production in $\overline{u^{\prime} w^{\prime}}$ is balanced largely by the pressure-strain term (Mansour, Kim \& Moin 1988; Pope 2000). The mean convection, turbulent transport, and pressure transport terms act to redistribute the Reynolds stresses in space and each of the transport terms integrates to zero over the flow depth in two-dimensional channel flow. The viscous transport term is expected to be negligible away from boundaries compared to other transport mechanisms if the Reynolds number is large. In the wake of an aquatic plant patch, the time-averaged flow field is three-dimensional and some departure from the distributions of the budget terms for two-dimensional flow may be expected. Some of the terms in (4.1) cannot be evaluated from the experimental 
data. The pressure field is not available, terms involving transverse derivatives cannot be calculated, and there is insufficient spatial resolution to resolve the dissipation rate tensor that would require resolution of the order of the Kolmogorov microscale $(\sim 0.1 \mathrm{~mm})$. We can, however, estimate contributions from streamwise and vertical derivatives to the mean convection, turbulent transport, and production terms as

$$
\begin{aligned}
& \left.\begin{array}{ll}
\text { mean conv. } & -\rho \bar{u} \frac{\partial \overline{\overline{u[1]}^{\prime} u_{[2]}}}{\partial x}-\rho \bar{w} \frac{\partial \overline{u_{[1]^{\prime} u_{[2]}}}}{\partial z}-\left(\rho \bar{v} \frac{\partial \overline{u^{\prime} u^{\prime}}}{\partial y}\right), \\
\text { turb. trans. } & -\rho \frac{\partial \overline{u^{\prime} u^{\prime} u^{\prime}}}{\partial x}-\rho \frac{\partial \overline{w^{\prime} u^{\prime} u^{\prime}}}{\partial z}-\left(\rho \frac{\partial \overline{v^{\prime} u^{\prime} u^{\prime}}}{\partial y}\right), \\
\text { production } & -2 \rho \overline{u_{[1]} u_{[2]}} \frac{\partial \bar{u}}{\partial x}-2 \rho \overline{u^{\prime} w^{\prime}} \frac{\partial \bar{u}}{\partial z}-\left(2 \rho \overline{u^{\prime} v^{\prime}} \frac{\partial \bar{u}}{\partial y}\right),
\end{array}\right\} \overline{\bar{u}^{\prime} u^{\prime}} \text { budget, } \\
& \text { mean conv. } \quad-\rho \bar{u} \frac{\partial \overline{v^{\prime} v^{\prime}}}{\partial x}-\rho \bar{w} \frac{\partial \overline{v^{\prime} v^{\prime}}}{\partial z}-\left(\rho \bar{v} \frac{\partial \overline{v^{\prime} v^{\prime}}}{\partial y}\right), \\
& \text { turb. trans. } \quad-\rho \frac{\partial \overline{u^{\prime} v^{\prime} v^{\prime}}}{\partial x}-\rho \frac{\partial \overline{w^{\prime} v^{\prime} v^{\prime}}}{\partial z}-\left(\rho \frac{\partial \overline{v^{\prime} v^{\prime} v^{\prime}}}{\partial y}\right), \quad\left\{\overline{v^{\prime} v^{\prime}}\right. \text { budget, } \\
& \text { production } \quad-2 \rho \overline{u^{\prime} v^{\prime}} \frac{\partial \bar{v}}{\partial x}-2 \rho \overline{v^{\prime} w^{\prime}} \frac{\partial \bar{v}}{\partial z}-\left(2 \rho \overline{v^{\prime} v^{\prime}} \frac{\partial \bar{v}}{\partial y}\right), \\
& \text { mean conv. } \quad-\rho \bar{u} \frac{\partial \overline{w^{\prime} w^{\prime}}}{\partial x}-\rho \bar{w} \frac{\partial \overline{w^{\prime} w^{\prime}}}{\partial z}-\left(\rho \bar{v} \frac{\partial \overline{w^{\prime} w^{\prime}}}{\partial y}\right), \\
& \text { turb. trans. } \left.\quad-\rho \frac{\partial \overline{u^{\prime} w^{\prime} w^{\prime}}}{\partial x}-\rho \frac{\partial \overline{w^{\prime} w^{\prime} w^{\prime}}}{\partial z}-\left(\rho \frac{\partial \overline{v^{\prime} w^{\prime} w^{\prime}}}{\partial y}\right), \quad\right\} \overline{w^{\prime} w^{\prime}} \text { budget, } \\
& -2 \rho \overline{u^{\prime} w^{\prime}} \frac{\partial \bar{w}}{\partial x}-2 \rho \overline{w^{\prime} w^{\prime}} \frac{\partial \bar{w}}{\partial z}-\left(2 \rho \overline{v^{\prime} w^{\prime}} \frac{\partial \bar{w}}{\partial y}\right),
\end{aligned}
$$

mean conv. $\quad-\rho \bar{u} \frac{\partial \overline{\bar{u}^{\prime} w^{\prime}}}{\partial x}-\rho \bar{w} \frac{\partial \overline{\bar{u}^{\prime} w^{\prime}}}{\partial z}-\left(\rho \bar{v} \frac{\partial \overline{u^{\prime} w^{\prime}}}{\partial y}\right)$,

turb. trans. $\quad-\rho \frac{\partial \overline{u^{\prime} u^{\prime} w^{\prime}}}{\partial x}-\rho \frac{\partial \overline{u^{\prime} w^{\prime} w^{\prime}}}{\partial z}-\left(\rho \frac{\partial \overline{u^{\prime} w^{\prime} v^{\prime}}}{\partial y}\right)$,

production

$$
\begin{aligned}
& -\rho \overline{u^{\prime} w^{\prime}} \frac{\partial \bar{u}}{\partial x}-\rho \overline{w^{\prime} w^{\prime}} \frac{\partial \bar{u}}{\partial z}-\rho \overline{u_{[1]} u_{[2]}} \frac{\partial \bar{w}}{\partial x}-\rho \overline{u^{\prime} w^{\prime}} \frac{\partial \bar{w}}{\partial z} \\
& -\left(\rho \overline{w^{\prime} v^{\prime}} \frac{\partial \bar{u}}{\partial y}-\rho \overline{u^{\prime} v^{\prime}} \frac{\partial \bar{w}}{\partial y}\right),
\end{aligned}
$$

$\rho \overline{u^{\prime} w^{\prime}}$ budget, (4.5)

in where the terms in brackets highlight the transverse derivatives that could not be calculated in this study. The effect of random measurement errors should be negligible for the terms involving third moments (all turbulent transport terms) and terms involving the fluid stresses $\overline{u_{[1]^{\prime} u_{[2]}}}, \overline{u^{\prime} w^{\prime}}, \overline{u^{\prime} v^{\prime}}$, or $\overline{v^{\prime} w^{\prime}}$. Terms involving $\overline{v^{\prime} v^{\prime}}$ or $\overline{w^{\prime} w^{\prime}}$ will be biased by the measurement noise, but evaluation of the magnitude of the noise contribution to each of these terms suggests that in all cases it is much smaller than the sampling error. Sampling errors were estimated using a resampling technique (Garcia, Jackson \& Garcia 2006) and associated confidence intervals are indicated in figure 9. In general, the sampling error varies with $z$, but in order to 
(a)

Measurement porosity $\overline{\phi_{M}}$

$(x=350 \mathrm{~mm})$

Reynolds stress

Kurtosis

$\rho \overline{u^{\prime} u^{\prime}}$ budget

$\rho \overline{v^{\prime} v^{\prime}}$ budget

$\rho \overline{u^{\prime} w^{\prime}}$ budget

Convection velocity
0.75

0.50

0.25

0
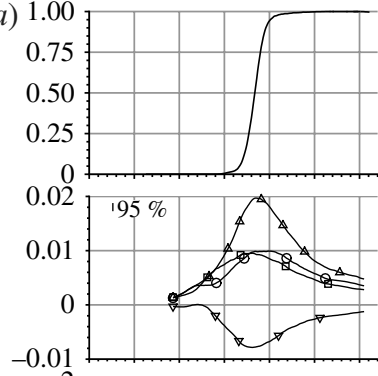

$-0.01$

Skewness

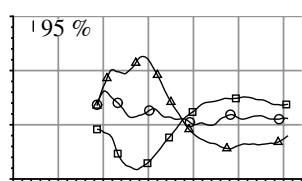

$$
-1
$$

b)
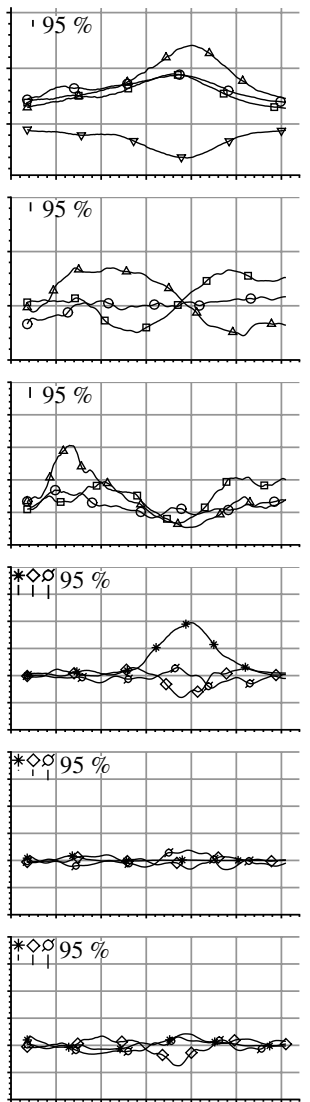

楼 $195 \%$
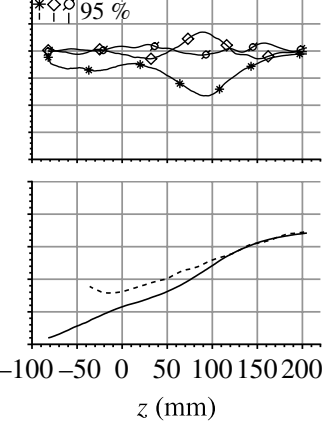

0.2

0
$-100-50 \quad 0 \quad 50100150200$

$z(\mathrm{~mm})$ $\triangle \overline{u_{[1]^{\prime} u_{[2]}}}$

$-\overline{v^{\prime} v^{\prime}}$

$\rightarrow \overline{w^{\prime} w^{\prime}}\left(\mathrm{m}^{2} \mathrm{~s}^{-2}\right)$

$\longrightarrow \overline{u^{\prime} w^{\prime}}$

$\unlhd S_{u}$

$\multimap S_{v}$

$\longrightarrow S_{w}$

$\triangle K_{u}$

$\multimap K_{v}$

$\square-K_{w}$

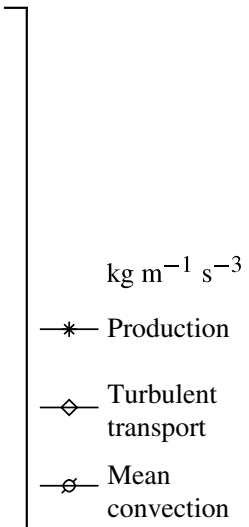

$\langle\bar{u}\rangle$

$\mathrm{m} \mathrm{s}^{-1}$

FIGURE 9. Velocity field statistics of a Ranunculus penicillatus plant patch: (a) the near wake, $x=430 \mathrm{~mm}$ (except measurement porosity, which is at $x=350 \mathrm{~mm}$ ); (b) the far wake $x=600 \mathrm{~mm}$. Error bars indicate approximate $95 \%$ confidence intervals.

reduce the clutter in figure 9, an average value is given. Derivatives were estimated by convolving the time-averaged moments of the velocity field with a $21 \times 21$ grid- 
point $(21 \mathrm{~mm} \times 21 \mathrm{~mm})$ second-order least-squares kernel. The size of the filter was sufficiently large to smooth over sampling errors (due to finite measurement duration), but still sufficiently small so as not to significantly reduce the amplitude of the measured derivatives.

Distributions of the available Reynolds stress budget terms together with the Reynolds stresses are shown in figure 9 for the near wake $(x=430 \mathrm{~mm})$ and for the far wake $(x=600 \mathrm{~mm})$. The distribution of the production term in the $\overline{u^{\prime} u^{\prime}}$ budget forms a peak at $z=85 \mathrm{~mm}$ in the near wake and $z=99 \mathrm{~mm}$ in the far wake which closely matches the peaks in the corresponding Reynolds normal stress. The mean convection term is also a gain at this elevation but is much smaller than the production term. The ratio between the local production and the mean convection terms, 3.8 in the near wake, suggests that the $\overline{u^{\prime} u^{\prime}}$ field near the shear layer is dominated by local rather than upstream generation processes. This result is consistent with a plane mixing layer (Wygnanski \& Fiedler 1970), but differs from many separated flows which feature a region where convection is the dominant gain term, for example the axisymmetric wake (Uberoi \& Freymuth 1970) and the wake of a surface-mounted cube (Hussein \& Martinuzzi 1996). The turbulent transport term in the $\overline{u^{\prime} u^{\prime}}$ budget is a loss near the shear layer and a gain at both higher and lower elevations. The effect of this term is therefore to diffuse turbulence away from the shear layer where it is produced. It is interesting to note that both turbulent transport and mean convection terms cross zero above and below the shear layer at about the same elevations $(z=114 \mathrm{~mm}$ and $z=64 \mathrm{~mm}$ respectively). Further, these elevations correspond to measurement porosity values measured at $x=350 \mathrm{~mm}$ of $\overline{\phi_{M}}=0.98$ and 0.04 respectively, i.e. near the extreme upper and lower elevations of the top of the fluctuating plant (considering that $\overline{\phi_{M}}$ in this region is dominated by the presence or absence of plant within PIV interrogation regions). The alignment between these three statistics may indicate that the fluctuating plant (and corresponding fluctuation of the shear layer elevation) plays a role in regulating the distribution of $\overline{u^{\prime} u^{\prime}}$ in the wake. Production in the $\overline{v^{\prime} v^{\prime}}$ and $\overline{w^{\prime} w^{\prime}}$ budgets is small compared to the production in the $\overline{u^{\prime} u^{\prime}}$ budget and does not appear to explain why the maxima in the three Reynolds normal stress distributions do not coincide. The reason for this is likely to be contained in the pressure-strain and pressure transport terms (which are not available from experimental data) and also in upstream production such as in the wakes of individual plant stems and leaves. Both the turbulent transport and mean convection terms in the transverse and vertical normal stress budgets have similar characteristics to the corresponding terms in the streamwise normal stress budget. The transport terms are a loss where the velocity variance is high and a gain in both the higher and lower flow layers. Convection terms follow the same pattern, but are smaller and have opposite sign. The $\overline{u^{\prime} w^{\prime}}$ budget has similar characteristics to the $\overline{u^{\prime} u^{\prime}}$ budget, but each term has opposite sign because the primary Reynolds shear stress is negative. We note again a correlation between the distribution of the production term and the corresponding Reynolds stress distribution with the local peaks in these distributions forming at the same elevation. The production is 7.3 times larger than the convection term indicating that the primary Reynolds shear stress distribution is dominated by local rather than upstream production. The turbulent transport and mean convection terms have opposite signs and, similar to the $\overline{u^{\prime} u^{\prime}}$ budget, each crosses zero near the same elevation. 


\subsection{Higher-order moments}

Skewness $S_{i}=\overline{u_{i}^{\prime} u_{i}^{\prime} u_{i}^{\prime}} / \overline{u_{i}^{\prime} u_{i}^{\prime}}{ }_{i}^{3 / 2}$ and kurtosis $K_{i}=\overline{u_{i}^{\prime} u_{i}^{\prime} u_{i}^{\prime} u_{i}^{\prime}} / \overline{u_{i}^{\prime} u_{i}^{\prime}}{ }^{2}-3$ (repeated index does not imply summation) distributions provide further indication of the nature of the turbulence in the wake of the plant patch. Equations (2.19)-(2.22) indicate that measured skewness and kurtosis are biased towards zero by the measurement noise. The relative error in $S_{w}$ is around $10 \%$ and in $K_{w}$ is around $15 \%$ in the shear zone behind the plant patch. Relative errors for $S_{v}$ and $K_{v}$ are $3 \%$ and $4 \%$ respectively and for $S_{u}$ and $K_{u}$ the error contribution is minimized using (2.17) and (2.18). Skewness is an indicator of the asymmetry of the velocity probability distribution, with negative skewness associated with a left-tailed distribution (rare high-magnitude velocity fluctuations tend to have a negative sign) while positive skewness indicates a right-tailed distribution (rare high-magnitude events tend to have a positive sign). Figure 9 indicates that $S_{v}$ in the wake of the plant is near zero over much of the flow depth, which is expected due to the approximate symmetry of the time-averaged flow field near the plant centreline. Skewness of the streamwise and bed-normal velocity components has opposite signs over most of the flow depth. A transition from an 'ejection'-dominated upper flow region $\left(S_{u}<0, S_{w}>0\right)$ to a 'sweep'-dominated lower flow region $\left(S_{u}>0, S_{w}<0\right)$ is evident around $z=88 \mathrm{~mm}$ in the near wake, which corresponds to the location of the mean shear layer. Such antisymmetric distributions of $S_{u}$ and $S_{w}$ are typical of mixing layers and flows over aquatic canopies (Raupach et al. 1996; Nezu \& Sanjou 2008) but are also found in open channel flows over gravel beds (Nikora \& Goring 2000b). The kurtosis of a probability distribution is an indicator of its 'peakedness' relative to a Gaussian distribution. A high value of the kurtosis coefficient of a velocity signal indicates the presence of rare (intermittent) high-magnitude events, while a kurtosis coefficient that is less than zero indicates that high-magnitude events occur more frequently than for a Gaussian distribution. Figure 9 indicates that the kurtosis coefficient for each velocity component follows a similar distribution, with regions of positive kurtosis in the higher and lower flow layers and a region of low kurtosis near the shear layer. The largest values of kurtosis are found behind the plant for elevations between $z=0 \mathrm{~mm}$ and $z=50 \mathrm{~mm}$. This indicates, in conjunction with $S_{u}>0, S_{w}<0$, that the flow field in this region is characterized by rare high-magnitude sweep events that are likely to originate from higher flow layers and intermittently impinge into the low-velocity region behind the plant. The negative value of kurtosis near the mean shear layer $\left(K_{u}=-0.61\right.$ at $z=88 \mathrm{~mm}$ ) is similar to the value of -0.63 measured by Wygnanski \& Fiedler (1970) at the centre of a plane mixing layer. Negative values of kurtosis have also been found in the near-bed region of gravel-bed open channel flows by Nikora \& Goring (2000b) and for a smooth-wall boundary layer by Balachandar et al. (2001). It is interesting to note that in each of these examples, and also for the present aquatic plant wake, the location of minimum kurtosis corresponds to the location of maximum variance.

\subsection{Convection velocity}

Eddy convection velocity $\left(\overline{u_{c}}\right)$ has previously been studied primarily because of its relevance to Taylor's 'frozen turbulence' approximation which can be applied to transform velocity statistics (such as velocity spectra and correlation functions) between time and space domains. Several studies have indicated surprising departures of the convection velocity from the local mean velocity (with $\overline{u_{c}}>\bar{u}$ ) such as in terrestrial canopy flows by Shaw et al. (1995), in aquatic canopies by Nezu \& Sanjou (2008), and for gravel-bed open channel flows by Nikora \& Goring (2000a). 
Understanding the reasons for this departure may provide some further insight into the turbulence structure, and this is our motivation for examining the convection velocity in the wake of the Ranunculus plant patch.

Convection velocity in the wake of the plant patch can be estimated from the two-point space-time correlation:

$$
\begin{aligned}
& R\left(z, x_{u}, x_{d}, \Delta t_{n}\right)\left[\overline{u^{\prime} u^{\prime}}\left(x_{u}, z\right) \overline{u^{\prime} u^{\prime}}\left(x_{d}, z\right)\right]^{0.5} \\
& =\frac{\sum_{t_{n}=1}^{t_{n}=T}\left[u^{\prime}\left(x_{u}, z, t_{n}\right) u^{\prime}\left(x_{d}, z, t_{n}+\Delta t_{n}\right) \phi_{M}\left(x_{u}, z, t_{n}\right) \phi_{M}\left(x_{d}, z, t_{n}+\Delta t_{n}\right)\right]}{\sum_{t_{n}=1}^{t_{n}=T}\left[\phi_{M}\left(x_{u}, z, t_{n}\right) \phi_{M}\left(x_{d}, z, t_{n}+\Delta t_{n}\right)\right]},
\end{aligned}
$$

where $x_{u}$ and $x_{d}$ identify 'upstream' and 'downstream' $x$ coordinates, $t_{n}$ is the time step, $\Delta t_{n}$ is time step separation, $T$ is the total number of time steps, and $\phi_{M}$ is the measurement clipping function described in $\$ 2.2$. The eddy convection velocity is then:

$$
\overline{u_{c}}\left(z, x_{u}, x_{d}\right)=\frac{x_{d}-x_{u}}{\Delta t_{R_{\max }}} f_{s},
$$

where $\Delta t_{R_{\max }}$ is the time separation (measured by time steps) that maximizes $R$, and $f_{s}$ is the sampling frequency $(30 \mathrm{~Hz})$. The mean velocity field in the wake of the plant is not homogeneous, so in order to make a meaningful comparison between the convection velocity and the local mean velocity, the latter is spatially averaged over the range $x_{u}<x<x_{d}$ :

$$
\langle\bar{u}\rangle\left(z, x_{u}, x_{d}\right)=\frac{1}{\sum_{x=x_{u}} \sum_{t_{n}=1}^{x=x_{d}}\left[\phi_{M}\left(x, z, t_{n}\right)\right]} \sum_{x=x_{u}}^{x=x_{d}} \sum_{t_{n}=1}^{t_{n}=T}\left[u\left(x, z, t_{n}\right) \phi_{M}\left(x, z, t_{n}\right)\right] .
$$

Convection velocity and local average velocity are shown for the near wake $\left(x_{u}=410, x_{d}=450 \mathrm{~mm}\right)$ and for the far wake $\left(x_{u}=580, x_{d}=620 \mathrm{~mm}\right)$ in figure 9 . Below $z=115 \mathrm{~mm}$ in the near wake and below $z=120 \mathrm{~mm}$ in the far wake, the convection velocity deviates significantly from the local mean velocity. The result $\overline{u_{c}}>\langle\bar{u}\rangle$ might be expected in the lower flow layers $(z<50 \mathrm{~mm})$ as the turbulence in this region is characterized by rare high-magnitude velocity fluctuations which are generated near the shear layer (where the mean velocity is higher) and periodically impinge into the low-velocity region. It is reasonable to assume that these eddies propagate with a velocity close to the mean velocity where they are generated, explaining the observed $\overline{u_{c}}>\langle\bar{u}\rangle$ near the bed. We note, however, that near the shear layer $(z=85 \mathrm{~mm}$ in the near wake) where the velocity fluctuations are dominated by local production, we can still observe that the convection velocity is larger than the local mean velocity $\left(\overline{u_{c}} /\langle\bar{u}\rangle=1.2\right.$ at $\left.z=85 \mathrm{~mm}\right)$. Raupach et al. (1996) explain similar observations in terrestrial canopy flows by suggesting that eddies which dominate the two-point correlation $R$ are produced mainly during wind gusts and therefore naturally propagate with the higher velocity of the gust rather than the lower mean velocity. The relevance of this interaction mechanism between the outer flow and the shear layer eddies to the present experiment, where scale separation is much smaller, is not clear and remains to be clarified in future experiments. 


\subsection{Velocity spectra}

The structure of the velocity field in the wake of the plant is further examined by considering the power spectrum of velocity fluctuations, $F_{i i}(f)$. The spectrum can be evaluated for velocity data with missing samples using the Lomb-Scargle method (Lomb 1976; Scargle 1982) which can be written as:

$$
2 F_{i i}(\omega)=\frac{\left\{\sum_{t_{n}=1}^{t_{n}=T} u_{i} \phi_{M} \cos [\omega(t-\tau)]\right\}^{2}}{\sum_{t_{n}=1}^{t_{n}=T} \phi_{M} \cos ^{2}[\omega(t-\tau)]}+\frac{\left\{\sum_{t_{n}=1}^{t_{n}=T} u_{i} \phi_{M} \sin [\omega(t-\tau)]\right\}^{2}}{\sum_{t_{n}=1}^{t_{n}=T} \phi_{M} \sin ^{2}[\omega(t-\tau)]},
$$

where $t=t_{n} / f_{s}$ is the time corresponding to the $t_{n}$ th measurement sample, $\omega=2 \pi f$ is the angular frequency, $f$ is the linear frequency, and $\tau$ is a time lag adopted by Scargle (1982) to enforce invariance of the spectrum to time translation of the data and simplify the statistical behaviour, with:

$$
\tan (2 \omega \tau)=\frac{\sum_{t_{n}=1}^{t_{n}=T} \phi_{M} \sin [2 \omega t]}{\sum_{t_{n}=1}^{t_{n}=T} \phi_{M} \cos [2 \omega t]}
$$

The Lomb-Scargle method is equivalent to estimating the spectrum by a leastsquares fit of sine waves to the data and for regularly spaced data reduces to the conventional Fourier spectrum (Scargle 1982).

Comparison of the velocity power spectrum near the shear layer in the wake of the plant $(x=430, z=85 \mathrm{~mm}$, figure $10 a)$ with the spectrum at a higher elevation $(x=430, z=200 \mathrm{~mm}$, figure $10 b)$, where the influence of the plant is reduced, indicates a broad increase in energy across all resolved frequencies in the plant wake. Some flattening of the spectrum is evident at higher frequencies due to the contribution of aliasing and measurement noise. A subtle clustering of energy around $f=1 \mathrm{~Hz}$ can be seen in the wake spectrum, but there is no indication of a highly periodic component that would suggest a Kelvin-Helmholtz-type instability of the shear layer.

\subsection{Plant velocity fluctuations and plant-flow coupling}

The fluctuating movements of aquatic plants are important for several reasons. First, plant motion can enhance photosynthetic rate and nutrient uptake through increased delivery of light and nutrients to leaf surfaces (Koehl \& Alberte 1988; Nikora 2010). Second, plant drag forces (which determine plant survival during high flow periods) may be regulated, to some extent, by plant motion. There is some evidence that waving plants can experience less drag by aligning themselves with instantaneous velocity streamlines ('dynamic reconfiguration', Siniscalchi \& Nikora 2013), although in general the reverse may also be true. Finally, plant movement can enhance turbulent kinetic energy in the plant wake with implications for sediment transport and mixing processes. In the following, we study plant velocity fluctuations extracted from PIV images using the method described in $\S 2.3$, to examine the nature of the plant motion and potential interaction mechanisms with the turbulent flow. 

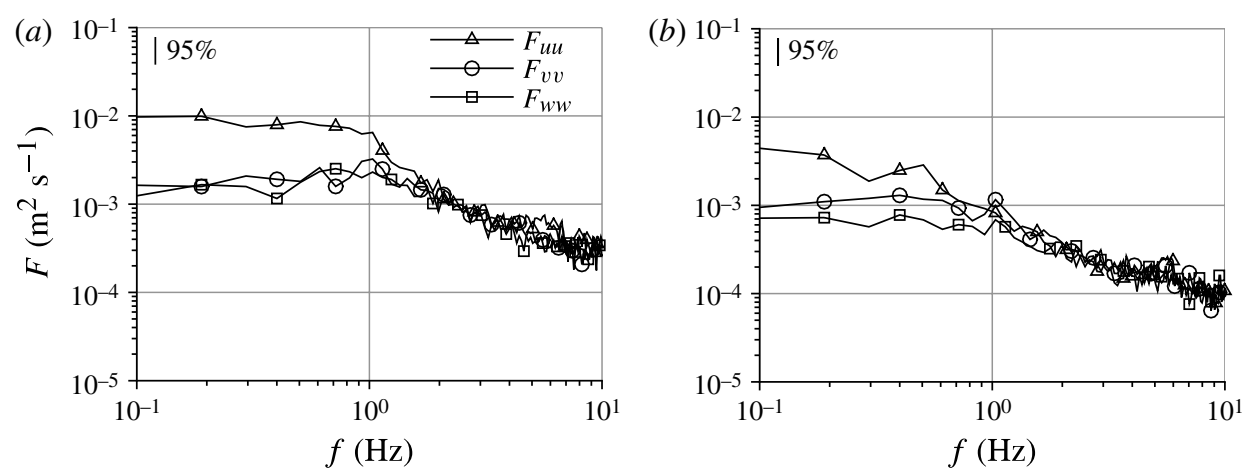

FIGURE 10. Flow velocity spectrum: $(a)$ behind the plant patch at $x=430, z=85 \mathrm{~mm}$; (b) above the plant patch at $x=430, z=200 \mathrm{~mm}$.

Measured vertical and transverse plant velocity variance (figure 11a) is found to increase rapidly approaching the free end of the plant, consistent with the similar measurements of Siniscalchi \& Nikora (2013) for a variety of aquatic plant species in a laboratory flume. The shape of the variance distribution reflects the structural dynamics of the plant and the turbulent forcing due to the fluctuating viscous and pressure stresses at plant surfaces. For simple structures undergoing free vibration (without external forcing) analytical solutions to the equations of motion may be obtained to predict the relative amplitude (and variance) of vibrations along the structure. The complex geometry of aquatic plants and their as yet uncertain biomechanical properties still preclude such analysis for the present case without dramatic simplifications. The ratio of transverse to vertical plant velocity variance is in the range 1.15-1.35 over the resolved plant length, quite similar to the corresponding ratio of fluid velocity variance in the wake of the plant (1-1.2). Further information about the nature of plant velocity fluctuations can be obtained from the two-point correlation function (4.6). Figure 11(b) indicates that the time $\left(\Delta t=f_{s}^{-1} \Delta t_{n}\right.$ ) corresponding to the maximum in the correlation function is increasing with increasing point separation $\left(\Delta x=x_{d}-x_{u}\right)$. This suggests that the characteristic plant motion is that of travelling waves rather than standing waves (vibration). These two phenomena are, however, closely related as standing waves can be considered to arise from the interference (constructive and destructive) of forward and backward propagating waves (Graff 1991). Païdoussis (2004), considering slender cylindrical structures aligned axially with the flow, indicates that wave propagation rather than vibration is typical for long structures. The propagation velocity estimated from the time lag that maximizes the correlation function (figure $11 b$ ) is $0.46 \mathrm{~m} \mathrm{~s}^{-1}$ for both $v_{p}{ }^{\prime}$ and $w_{p}{ }^{\prime}$, which is similar to the eddy convection velocity measured in the wake of the plant patch in the shear zone (figure 9). The similarity between these two convection velocities suggests that the waves propagating through the plant are dominated by the passage of turbulent fluctuations (vortices).

The plant velocity spectrum (figure $11 c, x=309 \mathrm{~mm}$ ) indicates maximum energy for frequencies around $1 \mathrm{~Hz}$ for both vertical and transverse components. The shape of the spectrum resembles that obtained in laboratory experiments using the same species of plant (Ranunculus penicillatus, Siniscalchi \& Nikora 2013) and features a significant decay of energy towards both lower and higher frequencies. In comparison, the transverse and vertical components of the fluid velocity spectrum measured outside 

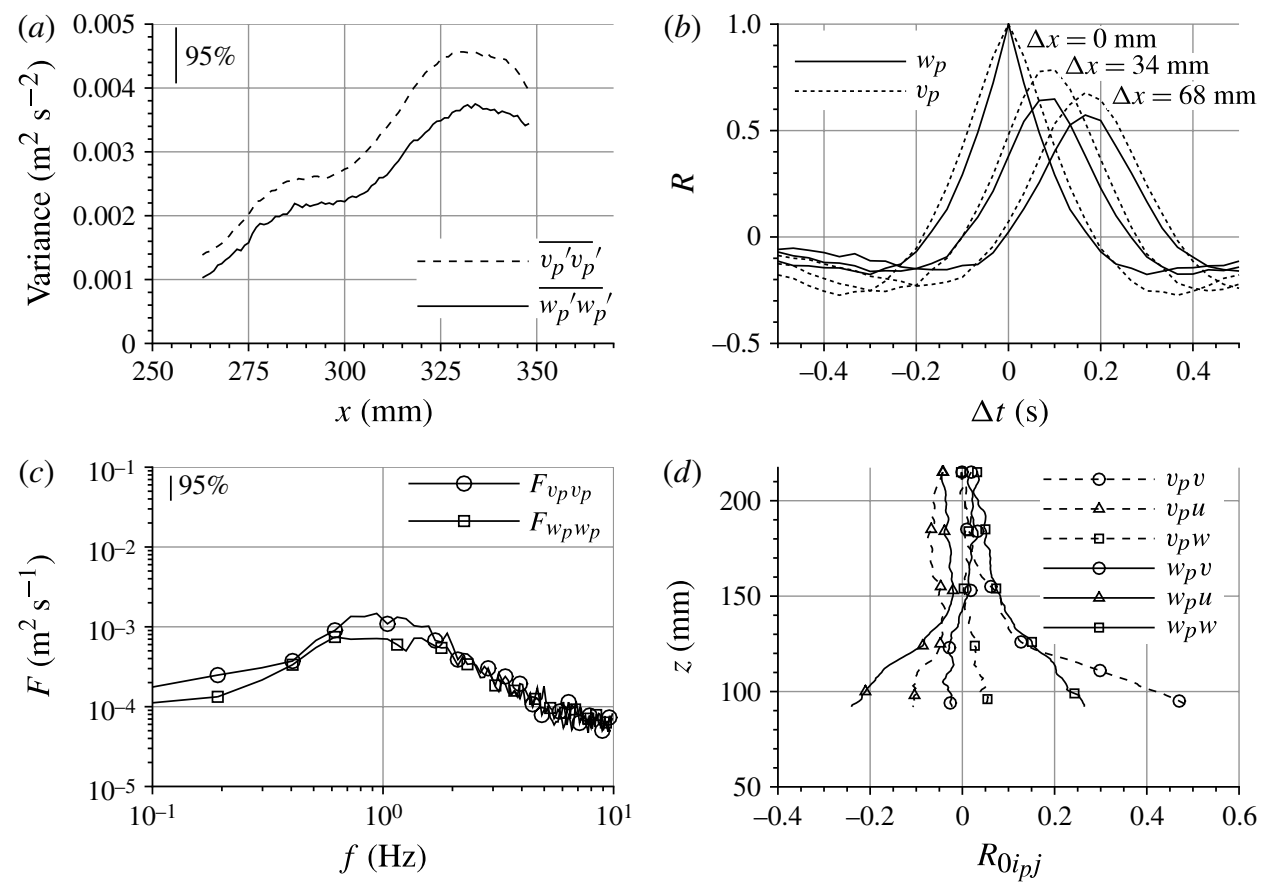

FIGURE 11. (a) Variation of plant velocity variance along the length of the plant. (b) Twopoint correlation of plant velocity fluctuations. (c) Spectrum of plant velocity fluctuations $(x=309 \mathrm{~mm})$. (d) Normalized covariance between plant and flow velocity fluctuations $(x=309 \mathrm{~mm})$.

the flow region influenced by the plant (figure 10b) are constant (saturated) for frequencies less than $1 \mathrm{~Hz}$. If the plant velocity can be considered as a (linearly) filtered response to the fluid velocity, figure $11(c)$ in comparison to figure $10(b)$ suggests that the plant responds optimally to frequencies around $1 \mathrm{~Hz}$ (or wavelengths $\bar{u} / f \sim 0.5 \mathrm{~m}$, i.e. of a similar scale to the patch length or flow depth). This observation may be related to Naudascher \& Rockwell's (1994) finding that for cylinders aligned axially with the flow, each vibration mode of the structure is most efficiently excited by vortices of a certain wavelength. Vibration modes for an aquatic plant are, however, yet to be identified. Possible mechanisms of flow-plant interaction are further discussed in $\S 5$.

Potential correlations between fluid $\left(u_{j}{ }^{\prime}\right)$ and plant $\left(u_{i p}{ }^{\prime}\right)$ velocity fluctuations can be further examined using the normalized covariance function:

$$
R_{0 i p j}(x, z)=\frac{\overline{u_{i p}{ }^{\prime}(x) u_{j}{ }^{\prime}(x, z)}}{\left(\overline{u_{i p}{ }^{\prime 2}} \overline{u_{j}{ }^{2}}\right)^{0.5}}
$$

with $i=2,3\left(v_{p}{ }^{\prime}, w_{p}{ }^{\prime}\right)$ and $j=1,2,3\left(u^{\prime}, v^{\prime}, w^{\prime}\right)$. For $x=309 \mathrm{~mm}$ and $z$ values approaching the free surface, the correlation between plant and fluid motion for all components is small $\left(R_{0 i_{p j}} \sim 0.05\right.$, figure $\left.11 d\right)$. The $R_{0 v_{p} u}, R_{0 v_{p} w}, R_{0 w_{p} v}$ terms remain small for all $z$, but the $R_{0 v_{p} v}, R_{0 w_{p} w}, R_{0 w_{p} u}$ terms increase rapidly approaching the top of the plant. While it is not surprising to find a correlation between matching velocity components $\left(R_{0 v_{p} v}, R_{0 w_{p} w}\right)$ and the cross-component term $R_{0 w_{p} u}$ through the 
secondary correlation $\overline{u^{\prime} w^{\prime}}<0$, the narrowness of the correlated range $\Delta z \sim 30 \mathrm{~mm}$ is unexpected. Given the $1 \mathrm{~Hz}$ characteristic frequency (figure 11c) of plant velocity fluctuations, we might reasonably be looking for characteristic eddy sizes of the order $\bar{u} / f \sim 0.5 \mathrm{~m}$ and a correspondingly larger correlation length. A supplementary movie showing a sequence of measured flow and plant velocity vectors and their relationship is available at http://dx.doi.org/10.1017/jfm.2013.406. In the following section we consider potential flow-plant interaction mechanisms that may help interpret the measured spectra and correlation functions.

\section{Flow-plant interactions: concluding remarks}

Naudascher \& Rockwell (1994) identified three general classes of flow-induced vibration mechanisms: extraneously induced excitation (EIE), instability-induced excitation (IIE), and movement-induced excitation (MIE). These classifications were developed to help identify and analyse the source of vibrations in engineering structures, but they are also relevant to the present case of flow-aquatic plant interactions, even if the characteristic plant motion is that of a propagating wave (figure $11 b$ ) rather than a vibration. Extraneously induced excitation relates to structure (plant) motion caused by turbulence in the flow, but independent of any local flow instability associated with the presence of the plant. Instability-induced excitation relates to motion induced by a flow instability that appears due to the presence of a structure. For an aquatic plant, this instability could, for example, be unsteady flow separation from the plant or a shear layer instability. Flow separation at a plant scale seems unlikely based on figure 6; however, there is significant turbulent kinetic energy associated with the shear layer in the wake of the plant patch (figures 8 and 9), and the short range of elevations over which plant and fluid velocity fluctuations are correlated (figure 11d) support a contribution of IIE to the plant motion. The absence of strong periodicity in the velocity spectrum measured in the plant patch wake (figure 10a), however, does not support an instability of the Kelvin-Helmholtz type. We did observe a weak clustering of energy around $1 \mathrm{~Hz}$ (matching well the dominant frequency of plant motion, figure 11c); however, this may simply reflect the flapping elevation of the shear layer as the plant moves up and down. The relative importance of EIE and IIE cannot be confirmed from the present experiments, but this could be further investigated in a laboratory environment by, for example, towing plants through stationary water to eliminate sources of EIE. Movement-induced excitation is a self-excited body vibration where the acceleration of a body in a fluid alters the flow field in a way that can feed back to the body (via pressure and viscous stresses) to amplify the initial movement. The 'flutter' of flags or aircraft wings are examples of MIE. The correspondence between measured convection velocities of plant velocity fluctuations and fluid velocity fluctuations in the plant patch wake suggests that the plant velocity fluctuations are dominated by the passage of turbulent eddies (either EIE and IIE), and MIE seems unlikely in the present case. Flexible cylinders aligned axially with the flow (resembling aquatic plant stems to some extent) can exhibit MIE at certain critical flow velocities, the dynamics for which have been studied extensively (e.g. Païdoussis 2004; de Langre et al. 2007). Extension of this type of analysis to an aquatic plant is not yet realistic due to the complex and changing plant geometry (reconfiguration) and the lack of fundamental knowledge of the coupling between fluid flow and resulting lift and drag forces acting on the plant.

Further experimentation in the laboratory and in the field is needed to clarify the nature of flow-plant interaction mechanisms. In this regard, we have demonstrated that 
the stereoscopic PIV method can be applied in field conditions and should prove to be valuable in further study of flow-aquatic plant interactions.

\section{Acknowledgements}

This research was partly supported by the Leverhulme Trust, grant F/00152/Z 'Biophysics of flow-plant interactions in aquatic systems' and was also stimulated by the Scientific Research Network WO.027p11N. The authors thank three anonymous reviewers for their helpful suggestions for improving this paper.

\section{Supplementary movie}

Supplementary movie are available at http://dx.doi.org/10.1017/jfm.2013.406.

\section{REFERENCES}

Albayrak, I., Nikora, V., Miler, O. \& O'Hare, M. 2012 Flow-plant interactions at a leaf scale: effects of leaf shape, serration, roughness and flexural rigidity. Aquat. Sci. 74 (2), 267-286.

Astarita, T. 2007 Analysis of weighting windows for image deformation methods in PIV. Exp. Fluids 43 (6), 859-872.

Balachandar, R., Blakely, D., Tachie, M. \& Putz, G. 2001 A study on turbulent boundary layers on a smooth flat plate in an open channel. Trans. ASME: J. Fluids Engng 123 (2), 394-400.

Bornette, G. \& Puijalon, S. 2011 Response of aquatic plants to abiotic factors: a review. Aquat. Sci. 73 (1), 1-14.

Calluaud, D. \& David, L. 2004 Stereoscopic particle image velocimetry measurements of the flow around a surface-mounted block. Exp. Fluids 36 (1), 53-61.

CAMERON, S. M. 2011 PIV algorithms for open-channel turbulence research: accuracy, resolution and limitations. J. Hydro-environ. Res. 5 (4), 247-262.

Cantwell, B. \& Coles, D. 1983 Experimental study of entrainment and transport in the turbulent near wake of a circular cylinder. J. Fluid Mech. 136, 321-374.

Davidson, P. A. 2004 Turbulence: An Introduction for Scientists and Engineers. Oxford University Press.

FOLKARD, A. M. 2005 Hydrodynamics of model Posidonia oceanica patches in shallow water. Limnol. Oceanogr. 50 (5), 1592-1600.

FOLKARD, A. M. $2011 a$ Flow regimes in gaps within stands of flexible vegetation: laboratory flume simulations. Environ. Fluid Mech. 11 (3), 289-306.

FolKard, A. M. $2011 b$ Vegetated flows in their environmental context: a review. Proc. Inst. Civil Engrs: Engng Comput. Mech. 164 (1), 3-24.

Garcia, C. M., JaCKSON, P. R. \& GARCia, M. H. 2006 Confidence intervals in the determination of turbulence parameters. Exp. Fluids 40 (4), 514-522.

Ghisalberti, M. \& NePF, H. M. 2002 Mixing layers and coherent structures in vegetated aquatic flows. J. Geophys. Res. C: Oceans 107 (2), 3-1.

GRAFF, K. F. 1991 Wave Motion in Elastic Solids. Dover.

Green, J. C. 2005 Velocity and turbulence distribution around lotic macrophytes. Aquat. Ecol. 39 (1-2), 1-10.

Hurther, D. \& LEMmin, U. 2001 A correction method for turbulence measurements with a 3D acoustic doppler velocity profiler. J. Atmos. Ocean. Technol. 18 (3), 446-458.

Hussein, H. J. \& MARTINUZZI, R. J. 1996 Energy balance for turbulent flow around a surface mounted cube placed in a channel. Phys. Fluids 8 (3), 764-780.

KATIJA, K. \& DABIRI, J. O. 2008 In situ field measurements of aquatic animal-fluid interactions using a self-contained underwater velocimetry apparatus (SCUVA). Limnol. Oceanogr.: Meth. 6 (April), 162-171. 
Koehl, M. A. R. \& Alberte, R. S. 1988 Flow, flapping, and photosynthesis of nereocystis leutkeana: a functional comparison of undulate and flat blade morphologies. Mar. Biol. 99 (3), 435-444.

DE LAngre, E. 2008 Effects of wind on plants. Annu. Rev. Fluid Mech. 40, 141-168.

De Langre, E., Païdoussis, M. P., Doaré, O. \& Modarres-SAdeghi, Y. 2007 Flutter of long flexible cylinders in axial flow. J. Fluid Mech. 571, 371-389.

Liao, Q., Bootsma, H. A., Xiao, J., Klump, J. V., Hume, A., Long, M. H. \& Berg, P. 2009 Development of an in situ underwater particle image velocimetry (UWPIV) system. Limnol. Oceanogr.: Meth. 7 (February), 169-184.

Lomb, N. R. 1976 Least-squares frequency analysis of unequally spaced data. Astrophys. Space Sci. 39 (2), 447-462.

MAAS, H. G. 1996 Contributions of digital photogrammetry to 3D PTV. In Three-dimensional Velocity and Vorticity Measuring and Image Analysis Techniques (ed. T. Dracos), pp. 191-208. Kluwer.

MAnsour, N. N., KIM, J. \& MoIN, P. 1988 Reynolds-stress and dissipation-rate budgets in a turbulent channel flow. J. Fluid Mech. 194, 15-44.

Naden, P., Rameshwaran, P., Mountford, O. \& Robertson, C. 2006 The influence of macrophyte growth, typical of eutrophic conditions, on river flow velocities and turbulence production. Hydrol. Process. 20 (18), 3915-3938.

Naudascher, E. \& Rockwell, D. 1994 Flow-Induced Vibrations: An Engineering Guide. Dover.

NEPF, H. M. 2012 Hydrodynamics of vegetated channels. J. Hydraul. Res. 50 (3), 262-279.

NezU, I. \& NaKagawa, H. 1993 Turbulence in Open Channel Flows. Balkema.

NeZU, I. \& SANJOU, M. 2008 Turburence structure and coherent motion in vegetated canopy open-channel flows. J. Hydro-environ. Res. 2 (2), 62-90.

NiKorA, V. 2010 Hydrodynamics of aquatic ecosystems: an interface between ecology, biomechanics and environmental fluid mechanics. River Res. Appl. 26 (4), 367-384.

Nikora, V., Cameron, S., Albayrak, I., Miler, O., Nikora, N., Siniscalchi, F., Stewart, M. \& O'HARE, M. 2012 Flow-biota interactions in aquatic systems: scales, mechanisms, and challenges. In Environmental Fluid Mecahanics: Memorial Volume in Honour of Prof. Gerhard H. Jirka (ed. W. Rodi \& M. Uhlmann), pp. 217-235. CRC Press.

NiKORA, V. \& GoRING, D. G. $2000 a$ Eddy convection velocity and Taylor's hypothesis of 'frozen' turbulence in a rough-bed open-channel flow. J. Hydrosci. Hydraul. Engng 18 (2), 75-91.

Nikora, V. \& Goring, D. $2000 b$ Flow turbulence over fixed and weakly mobile gravel beds. J. Hydraul. Engng 126 (9), 679-690.

NikORA, N. \& NiKORA, V 2007 A viscous drag concept for flow resistance in vegetated channels. In Harmonizing the Demands of Art and Nature in Hydraulics: Proceedings of 32nd Congress of IAHR, Venice, Italy (CD-ROM).

Nimmo Smith, W. A. M., Atsavapranee, P., Katz, J. \& Osborn, T. R. 2002 PiV measurements in the bottom boundary layer of the coastal ocean. Exp. Fluids 33 (6), 962-971.

Nobach, H. \& BodensChatz, E. 2009 Limitations of accuracy in PIV due to individual variations of particle image intensities. Exp. Fluids 47 (1), 27-38.

O’Hare, M. T., Hutchinson, K. A. \& Clarke, R. T. 2007 The drag and reconfiguration experienced by five macrophytes from a lowland river. Aquat. Bot. 86 (3), 253-259.

PAÏDoussis, M. P. 2004 Fluid-Structure Interactions: Slender Structures and Axial Flow, vol. 2. Elsevier.

Pope, S. B. 2000 Turbulent Flows. Cambridge University Press.

PrasAd, A. K. 2000 Stereoscopic particle image velocimetry. Exp. Fluids 29 (2), 103-116.

Raffel, M., Willert, C. E., Wereley, S. T. \& Kompenhans, J. 2007 Particle Image Velocimetry: A Practical Guide. Springer.

RaupaCh, M. R., Finnigan, J. J. \& BRUnet, Y. 1996 Coherent eddies and turbulence in vegetation canopies: the mixing-layer analogy. Boundary Layer Meteorol. 78 (3-4), 351-382.

SAND-JENSEN, K. 2003 Drag and reconfiguration of freshwater macrophytes. Freshwat. Biol. 48 (2), 271-283. 
SAnd-Jensen, K. \& Mebus, J. R. 1996 Fine-scale patterns of water velocity within macrophyte patches in streams. Oikos 76 (1), 169-180.

SCARGLE, J. D. 1982 Studies in astronomical time series analysis. II. Statistical aspects of spectral analysis of unevenly spaced data. Astrophys. J. 263 (2), 835-853.

Shaw, R. H., Brunet, Y., Finnigan, J. J. \& Raupach, M. R. 1995 A wind tunnel study of air flow in waving wheat: two-point velocity statistics. Boundary Layer Meteorol. 76 (4), 349-376.

Siniscalchi, F. \& NikORA, V. I. 2012 Flow-plant interactions in open-channel flows: a comparative analysis of five freshwater plant species. Water Resour. Res. 48 (5).

SinisCAlCHI, F. \& NiKORA, V. I. 2013 Dynamic reconfiguration of aquatic plants and its interrelations with upstream turbulence and drag forces. J. Hydraul. Res. 51 (1).

Siniscalchi, F., Nikora, V. I. \& Aberle, J. 2012 Plant patch hydrodynamics in streams: mean flow, turbulence, and drag forces. Water Resour. Res. 48 (1).

Sukhodolova, T. A. \& Sukhodolov, A. N. 2012 Vegetated mixing layer around a finite-size patch of submerged plants: 1. Theory and field experiments. Water Resour. Res. 48 (10).

Tritico, H. M., Cotel, A. J. \& Clarke, J. N. 2007 Development, testing and demonstration of a portable submersible miniature particle imaging velocimetry device. Meas. Sci. Technol. 18 (8), 2555-2562.

Uberoi, M. S. \& Freymuth, P. 1970 Turbulent energy balance and spectra of the axisymmetric wake. Phys. Fluids 13 (9), 2205-2210.

Vogel, S. 1994 Life in Moving Fluids: The Physical Biology of Flow. Princeton University Press.

WERnET, M. P. 2005 Symmetric phase only filtering: a new paradigm for DPIV data processing. Meas. Sci. Technol. 16 (3), 601-618.

WiEnEKe, B. 2005 Stereo-PIV using self-calibration on particle images. Exp. Fluids 39 (2), 267-280.

WILLERT, C. 1997 Stereoscopic digital particle image velocimetry for application in wind tunnel flows. Meas. Sci. Technol. 8 (12), 1465-1479.

Wygnanski, I. \& Fiedler, H. E. 1970 Two-dimensional mixing region. J. Fluid Mech. 41 (2), 327-361.

Zhu, W., Van Hout, R., Luznik, L., Kang, H. S., Katz, J. \& Meneveau, C. 2006 A comparison of PIV measurements of canopy turbulence performed in the field and in a wind tunnel model. Exp. Fluids 41 (2), 309-318. 